\title{
Resonances for a Semi-Classical Schrödinger Operator Near a Non Trapping Energy Level
}

By

\author{
Michel RouleuX*
}

\begin{abstract}
We give an example of a short range potential $V$ on the real line that is dilation analytic at infinity, non trapping at energy $E>0$, but oscillating in the neighborhood of some points, so rapidly that the Schrödinger operator $P=-h^{2} \Delta+V$ shows a string of resonances near $E$ in the lower half plane when $h>0$ is small enough. The extended states behave as standing waves partially reflected off the bumps of $V$. Such a potential is the analogue of the Wigner-Von Neumann potential in the case of embedded eigenvalues.
\end{abstract}

\section{$\S 0 . \quad$ Introduction}

Let $V$ be a potential on $\mathbf{R}^{n}$ such that $V(-\Delta+1)^{-1}$ is compact, and consider, for $h>0$, the Schrödinger operator $P=-h^{2} \Delta+V$ on $L^{2}\left(\mathbf{R}^{n}\right)$. Then $\sigma_{\text {ess }}(P)=[0,+\infty[$. To fix the ideas, assume $V(x)$ goes to zero as $|x| \rightarrow \infty$. One would expect the discrete spectrum of $P$ to be negative, for a quantum particle with strictly positive energy would eventually escape to infinity through tunneling; the celebrated Wigner-Von Neumann example shows that this guess is wrong. Namely there exists a potential $V$ on the real line as above such that, for $h=1, P$ has an embedded eigenvalue $E=1$. The asymptotic behaviour of $V$ reads (see [ReSim]):

$$
V(x)=-8 \frac{\sin 2 x}{x}+\mathcal{O}\left(|x|^{-2}\right), \quad|x| \rightarrow \infty
$$

and the eigenfunction associated with $E=1$

Communicated by T. Kawai, October 18, 1993. Revised May 22, 1997 and February 9, 1998. 1991 Mathematics Subject Classification(s): 34L40, 81Q20

* Centre de Physique Théorique, CNRS Luminy, Case 907, 13288 Marseille Cedex 9, France and PHYMAT, Université de Toulon et du Var

Present address: Université Paris Sud, Dept. of Math., 91405 Orsay cedex, France 


$$
\psi(x)=\frac{\sin x}{x}\left(1+\mathcal{O}\left(|x|^{-2}\right)\right)
$$

behaves as a standing wave reflected coherently off the bumps of $V$.

(Of course, it should be pointed out that a coherence condition in the frequencies is not sufficient to get an eigenvalue, as shows the case of periodic potentials ; the decay of $V$ at infinity also plays a role).

Conversely, Froese and Herbst (see [CFKS]) showed that if $V(x)=\kappa_{0} \frac{\sin 2 x}{x}$ $+V_{1}(x)$, where $\kappa_{0} \in \mathbf{R}, \quad V_{1}(-\Delta+1)^{-1}$ and $(-\Delta+1)^{-1} x \cdot \nabla V_{1}(-\Delta+1)^{-1}$ are compact, then $P$ verifies a Mourre estimate at all positive energies but 0 and 1, which implies that $P$ has no (strictly) positive eigenvalue except possibly at $E=1$. In a recent paper [K1], Klaus gives asymptotics of the analytic continuation of the $S$-matrix near such a critical energy (see also $[\mathrm{Ku}]$ ).

Assume further that $V$ is dilation analytic at infinity, i.e. $V$ is smooth and extends analytically outside a compact set $K$ of $\mathbf{R}^{n}$ in a domain:

$$
\Gamma=\left\{x \in \mathbb{C}^{n}:|\operatorname{Im} x| \leq C(1+|\operatorname{Re} x|), \quad \operatorname{Re} x \in \mathbb{R}^{n} \backslash K\right\}
$$

for some $C>0$, where:

$$
\lim _{x \in \Gamma \cdot|x| \rightarrow \infty} V(x)=0
$$

Then $P$ has only continuous spectrum above 0 and we may define the resonances of $P$ near the energy level $E>0$ by the method of analytic distorsions of Hunziker [Hu]. In the semi-classical limit $(h \rightarrow 0)$ the existence of resonances relies very much on the underlying dynamical classical system. Namely, let $p(x, \xi)=\xi^{2}+V(x)$ be the classical hamiltonian. For $I=[E-\varepsilon, E+\varepsilon]$ define the outgoing and incoming tails:

$$
\Gamma_{ \pm}=\left\{(x, \xi) \in p^{-1}(I) \mid \exp t H_{p}(x, \xi) \rightarrow \infty \quad \text { as } t \rightarrow \mp \infty\right\}
$$

(here $H_{p}$ denotes the hamiltonian vector field) and $K(I)=\Gamma_{+} \cap \Gamma_{-}$as the set of trapped trajectories (see [Ge Sj]). We say that $V$ is non-trapping at energy $E$ if $K(I)=\emptyset$ for $\varepsilon>0$ small enough. Generically, if $V$ is non trapping at energy $E$, there are no resonances too close to the real axis near $E$; resonances free domains were extensively studied by many authors. Thus, if $V$ is everywhere analytic, there are no resonances in a $h$-independent neighborhood of $E$ in the lower half plane; if $V$ is only of Gevrey class $G^{s}(s>1)$ in some compact 
set of $\mathbf{R}^{n}$, but dilation analytic at infinity, there are no resonances in a box like $[E-\delta, E+\delta]-i\left[0, \delta h^{1-1 / s}\right]$ for some $\delta>0$. If $V$ is only $C^{\infty}$ in some compact set of $\mathbf{R}^{n}$, but dilation analytic at infinity, there are no resonances in $[E-\delta, E+\delta]-i[0,-\delta h \log h]$ (see [Ro] and references therein). Note that these results have a natural counterpart in the scattering by a non-trapping obstacle for the wave equation. Thus, if the obstacle has an analytic boundary, then there are only finitely many resonances inside any neighborhood of the real axis of the form $-\operatorname{Im} z \leq C<\operatorname{Re} z>^{1 / 3}$; if the boundary is $G^{s}, s>1$, then for any $s^{\prime}>s$ there are only finitely many resonances inside any neighborhood of the real axis of the form $-\operatorname{Im} z \leq C<\operatorname{Re} z>^{1 /\left(2 s^{\prime}+1\right)}$; at last if the boundary has $C^{\infty}$ boundary, then there are only finitely many resonances inside any $\operatorname{logarithmic}$ neighborhood $-\operatorname{Im} z \leq C \log \langle\operatorname{Re} z>$ of the real axis (see [Me1], [BaLeRa], [SjZw] and [LasLas].)

On the other hand we know many examples of resonances created by trapped rays for the classical hamiltonian flow. Among typical examples are the shape resonances ([CoDuKleSe], [HeSj], [HiSi], [Na1], .), the barrier top $([\mathrm{Sj}],[\mathrm{BrCoDu} 2],[\mathrm{Na} 2], \cdots)$ and the closed trajectory of hyperbolic type [GeSj]. The last example is a quantum analogue of the resonances which appear in wave scattering by convex bodies which has also received considerable attention (see [Ik1,2], [Ge], and [LaxPh], [PetSto], [Me2] for recent surveys). Thus, a single ray that bounces between two convex obstacles gives raise to finitely many strings of resonances (depending on the dimension.)

One can then address the following problem: is there any potential which would give raise to resonances below a non trapping energy (necessarily far enough from the real axis)? Of course, the Wigner-Von Neumann potential would not do, nor any of its natural semi-classical extensions, for it is not dilation analytic and is certainly trapping at the relevant energies. But we keep in mind the salient feature of this example, namely the creation of a standing wave pattern. This can also be achieved for resonances if part of the extended state is reflected coherently between some bumps of the potential, which nevertheless would not be sensitive to the classical hamiltonian flow; indeed, the virial condition holds near the resonance with real part $E$, that is, there exists $\delta>0$ such that $2(V(x)-E)+x \cdot \nabla V(x)>\delta, x \in \mathbf{R}$. Under this condition the resonances cannot be too close to the real axis. Our example relies on a certain (local) non analyticity of $V$, due to fast oscillations. 
Let $s>3, a>0$ ( $a$ has to be slightly adjusted in function of $h$ ), and

$$
V(x, h)=\sqrt{2 h / \pi} e^{-h^{-1 / s}}\left(e^{-x^{2} / 2 h} \cos x / h+e^{-(x-a)^{2} / 2 h} \cos (x-a) / h\right)
$$

We look for resonances of $P=-h^{2} \Delta+V(x, h)$ near $E=\frac{1}{4}$ (we could also introduce a coupling constant $\lambda \in \mathbf{R}$; our results for $P_{\lambda}=-h^{2} \Delta+\lambda V(x, h)$ hold with a good uniformity with respect to $\lambda$ ). Note that $V$ is analytic, but if we are also interested in the dependence in $h$, as $h \rightarrow 0$ we only have:

$$
\left|\partial_{x}^{j} V(x, h)\right| \leq C^{j+1}(j !)^{s}, \quad 0<h \leq 1, \quad x \text { near } 0 \text { and } a
$$

for some $C>0$, i.e. $V$ is uniformly in $G^{s}$ as $h \rightarrow 0$, near 0 and $a$, while $V$ verifies uniform Cauchy estimates away from these points. Then the result of [Ro] shows that we have to search for resonances with an imaginary part less than $\sim-h^{1-1 / s}$.

This is precisely the order of magnitude we shall obtain. Let $P=-h^{2} \Delta+V$ be as above. For $\xi$ in a complex neighborhood of $\frac{1}{2}$ (whose size will depend on $h$ ), we define $P(\xi)=P-\xi^{2}$ so that $\xi^{2} \in \sigma(P) \Leftrightarrow 0 \in \sigma(P(\xi))$. For $\theta>0$ small enough (but independent of $h$ ), let $\Gamma_{\theta}$ be a family of standard distorsions of $\mathbb{R}$ in the sense of $[\mathrm{Hu}]$, so that $P$ extends as a closed operator on $L^{2}\left(\Gamma_{\theta}\right)$ with domain $H^{2}\left(\Gamma_{\theta}\right)$, the usual Sobolev space, with norm $\|\psi\|_{2}=\|(-\Delta+1) \psi\|_{L^{2}\left(\Gamma_{\theta}\right)}$. The contour $\Gamma_{\theta}$ is parametrized by $x \mapsto x_{\theta}=x e^{i \theta(x)}$ so that $\theta(x)=0$ for $-b<x<a+b$, and $\theta(x)=\theta$ for $x<-2 b$ or $x>a+2 b$. Here $b>0$ will be also chosen small enough but independent of $h$ (the choice of $\Gamma_{\theta}$ is specified in beginning of Sect. 1). We still denote by $P(\xi)$ the distorted operator.

Theorem 0.1. Fix $s>3$ and $a_{0}>0$. For $\xi$ as above, let $\eta=2 \xi-1$. For any $C_{0}>0$, and $\varepsilon_{0}>0$ small enough, consider the set

$$
W_{h}=\left\{\eta \in \mathbf{C}: \operatorname{Im} \eta \leq 0,|\eta|<C_{0} h^{1-1 / s}, a \operatorname{Im} \eta+2 h^{1-1 / s}>-\varepsilon_{0} h^{1-1 / s}\right\} .
$$

Then, if $a=(2 m+1) h, m \in \mathbf{N}$ in such a way that $\left|a-a_{0}\right|<$ Const. $h$, there exists a discrete subset $\Omega_{h} \subset W_{h}$ such that, for $h>0$ small enough:

$1^{\circ}$ If $\eta \in W_{h} \backslash \Omega_{h}$, then $P(\xi): H^{2}\left(\Gamma_{\theta}\right) \rightarrow L^{2}\left(\Gamma_{\theta}\right)$ is bijective with bounded inverse.

$2^{\circ}$ If $\eta \in \Omega_{h}$, then $0 \in \sigma(P(\xi)), P(\xi)$ is Fredholm of index 0 and splits into a direct sum $P(\xi): F(\xi) \oplus\left(F^{\prime}(\xi) \cap H^{2}\left(\Gamma_{\theta}\right)\right) \rightarrow F(\xi) \oplus F^{\prime}(\xi)$, where $F(\xi)$ is finite dimensional, $F^{\prime}(\xi)$ is closed and $P(\xi): F^{\prime}(\xi) \cap H^{2}\left(\Gamma_{\theta}\right) \rightarrow F^{\prime}(\xi)$ is bijective with bounded inverse.

$3^{\circ}$ If $\eta \in \Omega_{h}$, then either $\operatorname{dim} F(\xi)=2$ and $\left.P(\xi)\right|_{F(\xi)}$ is nilpotent of order 2 (i.e. 
$\left.P(\xi)\right|_{F(\xi)} \neq 0,\left.P^{2}(\xi)\right|_{F(\xi)}=0$ and we say we have a double resonance) or there exists $\tilde{\eta} \in \Omega_{h}$, with $\tilde{\xi}=\frac{1}{2} \tilde{\eta}+\frac{1}{2}$ such that $0<|\eta-\tilde{\eta}|=\mathcal{O}\left(e^{-1^{1 / C h^{1 / s}}}\right), C>0$, and $\operatorname{dim} F(\xi)=\operatorname{dim} F(\tilde{\xi})=1, F(\xi) \cap F(\tilde{\xi})=0$ (we say then that we have splitting of resonances).

$4^{\circ}$ There exists a surjection f from $\Omega_{h}$ onto the set of the roots of the equation:

$$
2 \pi h e^{-\eta^{2} / h} e^{2 i a \xi / h} \gamma(\xi)=1
$$

in $W_{h}$, such that $f(\eta)-\eta=\mathcal{O}\left(e^{-1^{\prime} C h^{1 / s}}\right), C>0$, where $\gamma(\xi)=-\left(8 \pi h \xi^{2}\right)^{-1} e^{-2 h^{-1 / s}}$. Moreover, if $\eta_{k}$ is such a root, then

$$
\eta_{k}=h^{1-1 / s}\left(\frac{2 k \pi}{a} h^{1 / s}-\frac{2 i}{a}\right)+h \widetilde{\zeta}_{k}
$$

where $\tilde{\zeta}_{k} \sim \sum_{j=1}^{\infty} h^{\prime j} \zeta_{k, j}(h)$ in the sense of classical analytic symbols in $h^{\prime}=$ $h^{1-2 / s}$.

Let us make a few remarks.

$1^{\circ}$ The range of the eigenprojector $\Pi\left(\xi_{0}\right)$ associated with some $\eta_{0}=2 \xi_{0}-1 \in \Omega_{h}$ ("double" or "splitted" resonance) is spanned by functions exponentially close to $F_{+}(x)$ and $F_{-}(x)$ (see (2.4)). The frequency set of $F_{+}(x)$ (as defined in $[\mathrm{GuSt}])$, is concentrated near $\left[0, \infty\left[\times\left\{\frac{1}{2}\right\} \cup\left(\right.\right.\right.$ finite set); this of $F_{-}(x)$ near $]-\infty, a] \times\left\{-\frac{1}{2}\right\} \cup($ finite set $)$.

The number $2 a \xi$ in the phase factor $e^{2 i a \xi / h}$ of the quantization condition (0.1) can thus be interpreted as the classical (complex) action the particle acquires from commuting between 0 and $a$, with constant momentum $\xi$. The idea of the proof is to "quantize" this loop.

$2^{\circ}$ Because of symmetry, we believe that we have splitting of resonances rather than a double pole for the resolvent (by analogy with the self adjoint case). If it is the case, then this splitting has certainly something to do with the distance between the two components $\xi= \pm \frac{1}{2}$ of the energy surface.

$3^{\circ}$ This example may be extended to higher dimensions. For instance in $\mathbf{R}^{\mathbf{3}}$ (odd dimension are easier to tackle) one could take:

$$
V(x, a) \sim e^{-h^{-1 / s}} \sum_{j=0}^{2} e^{-\left(R^{j} x-a\right)^{2} / 2 h} \cos \left(R^{j} x-a\right) \omega / h
$$


where $a \in \mathbb{R}^{3} \backslash 0$ and $\omega$ is a unit vector not parallel to $0 a$, and $R$ is the rotation of angle $2 \pi / 3$ in the plane $(0, a, \omega)$.

$4^{\circ}$ The choice of a potential independent of $h$, i.e. replace for instance $V(x, h)$ by:

$$
V(x)=\int_{1}^{\infty} f(\lambda)\left(e^{-\lambda x^{2} / 2} \cos \lambda x+e^{-\lambda(x-a)^{2} / 2} \cos \lambda(x-a)\right) d \lambda
$$

with $f(\lambda)$ decaying as $e^{-\lambda^{1 / s}}$ as $\lambda \rightarrow \infty$ would presumably lead to the same kind of results, and by stationary phase arguments, the main contribution of this potential to resonances would come from $\lambda=h^{-1}$.

$5^{\circ}$ We conjecture that there are no other string of resonances near $E=\frac{1}{4}$ when dimension is 1 ; namely if we keep in mind the analogy with a closed trajectory as described above, we can infer from Bohr-Sommerfeld quantization rule that $2 a \xi$ is the only relevant action that is responsible for creation of resonances (and no multiple of $2 a \xi$ ). In other terms there will be no new resonance revealed by winding around the loop several times. (Compare with [Ge] for the multidimensional case; according to another convention made in that paper, resonances are located in the upperhalf plane $\operatorname{Im} \xi \geq 0$, which corresponds to the substitution $\theta \mapsto-\theta$ for the distorsion.) Note that we have found the first string of resonances (if several).

$6^{\circ}$ The proof we present here is straightforward but very tedious. Note that the quantization condition can be obtained (heuristically) as follows: let $u_{ \pm}(x)$ be the solution of $\left(P-\xi^{2}\right) u=0$ in $L^{2}\left(\Gamma_{\theta}\right)$ with $u_{ \pm} \sim e^{ \pm i x \xi / h}, x \rightarrow \pm \infty$; then $\xi^{2}$ is a resonance iff $W\left(u_{+}, u_{-}\right)=0$ where $W\left(u_{+}, u_{-}\right)$denotes the wronskian of $u_{+}$and $u_{-}$. To compute $W\left(u_{+}, u_{-}\right)$it suffices to know the asymptotics of $u_{+}$and $u_{-}$near a point between 0 and $a$. Let $G_{+}(\xi)=i(2 \xi h)^{-1} \chi(y<x) e^{i(x-y) \xi / h}$ and $G_{-}(\xi)=i(2 \xi h)^{-1} \chi(y>x) e^{-i(x-y) \xi / h}$ be the half Green kernels for $Q(\xi)=-h^{2} \Delta-\xi^{2}$. Put: $v_{-}(x)=e^{-i x \xi / h}$ $-G_{+}(\xi) V e^{-i \cdot \xi / h}(x)$ and $v_{+}(x)=e^{i x \xi / h}-G_{-}(\xi) V e^{i \cdot \xi / h}(x)$. For $\delta<x<a-\delta$ we have: $v_{+}(x)=e^{i x \xi / h}-c(h) e^{-i x \xi / h} e^{2 i a \xi / h} \alpha(a-x, \eta)+\mathcal{O}\left(e^{-1 / C h^{\prime}}\right)$ and $v_{-}(x)=$ $e^{-i x \xi / h}-c(h) e^{i x \xi / h} \alpha(x, \eta)+\mathcal{O}\left(e^{-1 / C h^{\prime}}\right)$. (See the definition of $\alpha(x, \eta)$ in the beginning of Sect. 1; here $\delta=h^{1 / s}, h^{\prime}=h^{1-2 / s}, c(h)=i(4 \xi h)^{-1} \sqrt{2 h / \pi} e^{-h^{-1 / s}}$.) Then computing the wronskian for $\delta<x<a-\delta$ we find $W\left(v_{+}, v_{-}\right)=\frac{2 i \xi}{h}(1$ $\left.-c^{2}(h) e^{2 i a \xi / h} e^{-\eta^{2} / h} 2 \pi h\right)+\mathcal{O}\left(e^{-1 / C h^{\prime}}\right)$ and the equation $W\left(v_{+}, v_{-}\right)=0$ gives 
precisely the quantization condition in the theorem. Unfortunately we have not been able to give this idea a rigourous form.

\section{Acknowledgements}

I wish to thank J. Sjöstrand for adressing this problem to me, and a referee for his careful reading of very tedious computations.

\section{Fredholm Alternative for $I-(G(\xi) V)^{2}$}

\section{$\S 1$. The Kernel}

Let $\xi$ be in a complex neighborhood of $\frac{1}{2}$, and $Q(\xi)=-h^{2} \Delta-\xi^{2}$ be the free hamiltonian. The Green kernel for $Q(\xi)$ is:

$$
G(x, y, \xi)=G(\xi)=i(2 \xi h)^{-1}\left(\chi(y<x) e^{i(x-y) \xi / h}+\chi(x<y) e^{-i(x-y) \xi / h}\right), \quad x, y \in \mathbf{R}
$$

where, here and hereafter, $\chi$ denotes the (sharp) characteristic function. (Among all arguments of the various functions we shall consider, we omit the variable $h$ which appears everywhere.) All quantities will depend holomorphically on $\xi$ in a neighborhood of $\frac{1}{2}$. First we specify the standard distorsion. Let $b>0$, and $t$ be a smooth function on $\mathbb{R}$ such that $t \equiv 1$ on $]-\infty,-2 b]$ $\cup[a+2 b,+\infty[, t \equiv 0$ on $[-b, a+b], t$ is strictly decreasing on $[-2 b,-b]$, strictly increasing on $[a+b, a+2 b]$. We put $x_{\theta}=x e^{i \theta t(x)}$, which we shall denote $x_{\theta}=x e^{i \theta(x)}$ for simplicity. Let also $\mathscr{A}$ be a subset of entire functions on $\mathbf{C}$ such that:

i) for any $\theta$ in a complex neighborhood of $\left[0, \theta_{0}\right],\left(x_{\theta}\right)^{\star}(\mathscr{A})$ is a dense subset of $L^{2}(\mathbf{R})$.

ii) for any $\psi \in \mathscr{A}, \theta \mapsto\left(x_{\theta}\right)^{\star} \psi=\psi \circ x_{\theta}$ is an analytic family for $\theta$ in a complex neighborhood of $\left[0, \theta_{0}\right]$. Such a choice is given by (see $[\mathrm{Hu}]$ ):

$$
\begin{gathered}
\mathscr{A}=\left\{\psi \text { entire: } \forall N, \exists C_{N}>0, \psi(x) \leq C_{N}\left((1+|\operatorname{Re} x|)^{-N}\right),\right. \\
\text { if }|\operatorname{Im} x|<C(1+|\operatorname{Re} x|) \text { for some } C>0\}
\end{gathered}
$$

We shall extend $G(x, y, \xi)$ to complex variables $x, y \in \Gamma_{\theta}$. For this purpose we can consider the action of $G(\xi)$ on $u \in \mathscr{A}$. Namely, for real $x$ and $y$, we have:

$$
G(\xi) u(x)=i(2 \xi h)^{-1}\left(\int_{-\infty}^{x} e^{i(x-y) \xi / h} u(y) d y+\int_{x}^{+\infty} e^{-i(x-y) \xi / h} u(y) d y\right), \quad u \in \mathscr{A}
$$

and this equality still makes sense for $x, y \in \Gamma_{\theta}, u \in \mathscr{A}$ provided $\operatorname{Im}\left(e^{i \theta} \xi\right) \geq 0$. It 
is also clear that for fixed $\theta>0, \xi \mapsto G(\xi)$ is an analytic family of bounded operators from $L^{2}\left(\Gamma_{\theta}\right)$ to $H^{2}\left(\Gamma_{\theta}\right)$ (the usual Sobolev space) for $\xi^{2}$ away from $e^{-2 i \theta} \mathbf{R}^{+}$(the essential spectrum of $-h^{2} \Delta$ acting on $L^{2}\left(\Gamma_{\theta}\right)$.) $G(\xi)$ is symmetric in the sense:

$$
\langle G(\xi) u, v\rangle=\langle u, G(\xi) v\rangle
$$

where $\langle\cdot, \cdot\rangle$ denotes the bilinear symmetric pairing:

$$
\langle u, v\rangle=\int_{\mathbb{R}}\left(x_{\theta}\right)^{\star}(u(x) v(x) d x)
$$

that we simply denote by:

$$
\langle u, v\rangle=\int(u(x) v(x) d x)_{\theta} .
$$

Let now $P(\xi)=Q(\xi)+V$. It is known that when $\theta>0, \xi \neq 0, P(\xi)$ is a closed operator $L^{2}\left(\Gamma_{\theta}\right)$ on with domain $H^{2}\left(\Gamma_{\theta}\right)$. We are interested in the values of $\xi$ such that $0 \in \sigma_{d}(P(\xi))$. For $0 \notin \sigma(P(\xi)) \cup \sigma(Q(\xi))$, iterating the first resolvent formula: $P(\xi)^{-1}=G(\xi)-G(\xi) V P(\xi)^{-1}$, we get:

$$
\left(I-(G(\xi) V)^{2}\right) P(\xi)^{-1}=(I-G(\xi) V) G(\xi)
$$

We will show below that for $\xi$ close to $\frac{1}{2}, I-(G(\xi) V)^{2}: H^{2}\left(\Gamma_{\theta}\right) \rightarrow H^{2}\left(\Gamma_{\theta}\right)$ is Fredholm and invertible except for a discrete set $\Omega_{h}^{\prime}$ of $\xi$ 's (see Proposition 1.8; here $\Omega_{h}^{\prime}$ corresponds to $\Omega_{h}$ through the mapping $\eta=2 \xi-1$.) So when $0 \notin \sigma(P(\xi)) \cup \sigma(Q(\xi))$ and $\xi \notin \Omega_{h}^{\prime}$,

$$
P(\xi)^{-1}=\left(I-(G(\xi) V)^{2}\right)^{-1} G(\xi)(I-V G(\xi)) .
$$

This shows in turn that the spectrum of $P$ near $\frac{1}{4}$ is precisely contained in $\left\{\xi^{2} \mid \xi \in \Omega_{h}^{\prime}\right\}$. In section II, we shall prove that this set is actually a set of resonances by computing the projector (2.1) associated to an element of $\Omega_{h}$.

The standard formula (1.1) provides a better approximation to the spectral radius of $P$; further it codifies or "quantizes" the loop we have mentionned above. Following [RiN] in a very concrete way, we approximate $(G(\xi) V)^{2}$ by a finite rank operator. We begin with computing $(G(\xi) V)^{2}$ in the real domain. Set $\eta=2 \xi-1, \eta^{\prime}=2 \xi+1$ and define the functions for real $x, y$ : 


$$
\begin{gathered}
\alpha(x, \tau)=\int_{-\infty}^{x} e^{-t^{2} / 2 h} e^{-i t \tau / h} d t, \quad \tau=\eta \text { or } \eta^{\prime} \\
\beta(x, y)=\chi(x<y) \int_{x}^{y} \tilde{V}(t) d t
\end{gathered}
$$

where:

$$
\tilde{V}(t)=2 e^{-t^{2} / 2 h} \cos t / h+2 e^{-(t-a)^{2} / 2 h} \cos (t-a) / h
$$

and:

$$
\begin{aligned}
& \psi_{1}(y)=e^{-y^{2} / 2 h}+e^{i a / h} e^{-(y-a)^{2} / 2 h} \\
& \psi_{2}(y)=e^{-i a / h} \psi_{1}(a-y)=e^{-y^{2} / 2 h}+e^{-i a / h} e^{-(y-a)^{2} / 2 h} \\
& \varphi_{1}(y)=\alpha\left(y, \eta^{\prime}\right)+\alpha(y, \eta)+e^{-2 i a \xi / h}\left(\alpha\left(y-a, \eta^{\prime}\right)+\alpha(y-a, \eta)\right) \\
& \varphi_{2}(y)=e^{2 i a \xi / h} \varphi_{1}(a-y)=\alpha\left(-y, \eta^{\prime}\right)+\alpha(-y, \eta)+e^{2 i a \xi / h}\left(\alpha\left(a-y, \eta^{\prime}\right)+\alpha(a-y, \eta)\right)
\end{aligned}
$$

The frequency set of $\alpha(x, \eta)$ is concentrated near $[0,+\infty[\times\{0\}$; this function can be considered as a smoothed characteristic function of $[0,+\infty$ [(up to a normalization constant.) On the other hand, $\alpha\left(x, \eta^{\prime}\right)$ is oscillating and its frequency set is concentrated near $\{(0,-2)\}$. To estimate $\alpha(x, \tau)$, it will be convenient to use the following formula : let $F(t)=\frac{1}{2}\left(1+(2 \pi h)^{-1 / 2} \int_{-t}^{t} e^{-y^{2} / 2 h} d y\right)$. Then for all $t, \tau \in \mathbf{C}$ :

$$
e^{\tau^{2} / 2 h} \alpha(t, \tau)=\sqrt{2 \pi h} F(t-\operatorname{Im} \tau)+i e^{-(t-\operatorname{Im} \tau)^{2} / 2 h} \int_{0}^{\operatorname{Re} \tau} e^{y^{2} / 2 h} e^{-i y(t-\operatorname{Im} \tau) / h} d y
$$

We set also:

$$
\begin{aligned}
& \psi_{+}(y)=e^{i y \eta / h} \psi_{1}(y)+e^{i y \eta^{\prime} / h} \psi_{2}(y) \\
& \psi_{-}(y)=e^{-i y \eta / h} \psi_{2}(y)+e^{-i y \eta^{\prime} / h} \psi_{1}(y)
\end{aligned}
$$

that is,

$$
\psi_{-}(y)=e^{-2 i a \xi / h} \psi_{+}(a-y) .
$$

Let $\gamma(\xi)=-\left(8 \pi h \xi^{2}\right)^{-1} e^{-2 h^{-1 / s}}$ be as defined in Theorem $0 \cdot 1-4^{\circ}$, and put as usual for real $x$ and $y: x \wedge y=\min (x, y), x \vee y=\max (x, y)$. After some straightforward computation, we find: 


$$
(G(\xi) V)^{2}=\gamma(\xi) K(\xi)+\gamma(\xi) K^{\prime}(\xi)
$$

with:

$$
\begin{aligned}
& K(\xi)=e^{i(x-y) \xi / h} \psi_{+}(y) \varphi_{1}(x \wedge y)+e^{-i(x-y) \xi / h} \psi_{-}(y) \varphi_{2}(x \vee y) \\
& K^{\prime}(\xi)=e^{i(x+y) \xi / h} \psi_{-}(y) \beta(y, x)+e^{-i(x+y) \xi / h} \psi_{+}(y) \beta(x, y)
\end{aligned}
$$

(we have identified an integral operator with its kernel.) Let us make a few comments on the kernels $K(\xi)$ and $K^{\prime}(\xi)$ which might help to understand the interference pattern, at least on a heuristic level. It is easy to see, by stationary phase arguments (at least after reading the Appendix) that $K(\xi)$ roughly takes a function outgoing at $+\infty$ and whose frequency set is concentrated on $\left[0,+\infty\left[\times\left\{\frac{1}{2}\right\}\right.\right.$ (we can check this for $e^{i y \xi / h} \alpha(y, \eta)$ ), into a function which is also outgoing at $+\infty$ and whose frequency set is concentrated near $\left[0,+\infty\left[\times\left\{\frac{1}{2}\right\} \cup\right.\right.$ (finite set). In the same way, $K(\xi)$ roughly takes a function outgoing at $-\infty$ and whose frequency set is concentrated on $]-\infty, a] \times\left\{\frac{-1}{2}\right\}$ into a function whose frequency set is concentrated near $]-\infty, a] \times\left\{\frac{-1}{2}\right\} \cup$ (finite set). So we can say, somewhat loosely, that $K(\xi)$ essentially preserves each of the frequency sets $\left[0,+\infty\left[\times\left\{\frac{1}{2}\right\}\right.\right.$ and $\left.]-\infty, a\right] \times\left\{\frac{-1}{2}\right\}$ (modulo a finite set in phase space). So $K(\xi)$ set up constructive interferences at frequencies $\pm \frac{1}{2}$. The action of operator $K^{\prime}(\xi)$ is somewhat more difficult to describe (it exchanges incoming with outgoing waves,) but it will be treated mainly as a perturbation.

Again, the kernels $K(\xi)$ and $K^{\prime}(\xi)$ can be extended to $x, y \in \Gamma_{\theta}$. For instance $\beta(x, y)$ can be viewed as a kernel:

$$
\beta(x, \cdot) u(x)=\int_{x}^{+\infty} d y u(y) \int_{x}^{y} \tilde{V}(t) d t, \quad u \in \mathscr{A}
$$

where the integration is performed along $\Gamma_{\theta}$, with $x \in \Gamma_{\theta}$. Similarly, $\alpha(x \wedge y, \tau)$ denotes the kernel

$$
\alpha(x \wedge \cdot, \tau) u(x)=\int_{-\infty}^{x} e^{-t^{2} / 2 h} e^{-i t \tau / h} d t \int_{t}^{\infty} u(y) d y, \quad u \in \mathscr{A}
$$


which can be written also in the more convenient form:

$$
\alpha(x \wedge \cdot, \tau) u(x)=\int_{\gamma_{\wedge}(x)}\left(e^{-t^{2} / 2 h} e^{-i t \tau / h} u(y) d t \wedge d y\right)_{\theta}
$$

where, for $x \in \Gamma_{\theta}, \gamma_{\wedge}(x)=\left\{(t, y) \in \Gamma_{\theta} \mid t \prec y\right.$ and $\left.t \prec x\right\}$ and $\prec$ means: put on increasing order on $\Gamma_{\theta}$. Here we have ordered $\Gamma_{\theta}$ with the natural order on $\mathbf{R}$ induced by the injective map $x \mapsto x_{\theta}$. In the sequel we will often identify $\Gamma_{\theta}$ with $\mathbf{R}$, and carry out the computations exactly as if the variables were real, dropping the indices $\theta$.

We now explain how to replace $K(\xi)$ by a finite rank operator $T(\xi)$, modulo a remainder term $H^{\prime}(\xi)$. This will be the starting point when applying Fredholm theory to $I-(G(\xi) V)^{2}$, the errors $\gamma(\xi) H^{\prime}(\xi)$ and $\gamma(\xi) K^{\prime}(\xi)$ being corrected by a Neumann series. From the expression of $K(\xi)$ we see that away from the diagonal $x=y$ the kernel of this operator is a simple tensor product. On the other hand the functions $\psi_{ \pm}(y)$ decay rapidly outside $y=0$ or $y=a$.

To be more precise, let $h^{\prime}=h^{1-2 / s}$. We shall work modulo exponentially small errors, whose order of magnitude is either $\exp \left(-1 / C h^{1 / 5}\right), \exp \left(-1 / C h^{\prime}\right)$ or $\exp (-1 / C h)(C>0)$. The $\exp (-1 / C h)$ terms are completely negligible, but when $s>3$, the $\exp \left(-1 / C h^{\prime}\right)$ errors are also very small; namely for any quantity $f(h)$ of temperate growth in the scales $\exp \left(c / h^{1 / s}\right), c>0$, i.e. $f(h)=\mathcal{O}\left(\exp \left(c / h^{1 / s}\right)\right)$, $h \rightarrow 0$, we have $\exp \left(-1 / C h^{\prime}\right) f(h)=\mathcal{O}\left(\exp -1 / C^{\prime} h^{\prime}\right)$ for some $C^{\prime}>0$ when $h>0$ is small enough.

So let $\delta=h^{1 / s}$. The functions $\psi_{ \pm}(y)$ behave like $e^{-y^{2} / 2 h}$ or $e^{-(y-a)^{2} / 2 h}$ and $\psi_{ \pm}(y)=\mathcal{O}\left(\exp \left(-1 / C h^{\prime}\right)\right)$ outside $y=0$ and $y=a$. So we can remove from $K(\xi)$ (as it will be stated precisely below, the contributions of the domains $(x<-\delta) \cap(y<-\delta),(\delta<x<a-\delta) \cap(\delta<y<a-\delta)$ and $(a+\delta<x) \cap(a+\delta<y)$ surrounding the diagonal. The remaining part close to the diagonal is then $(-\delta<x<\delta) \cap(-\delta<y<\delta)$ and $(a-\delta<x<a+\delta) \cap(a-\delta<y<a+\delta)$. There, by (1.2) we have respectively:

$$
\begin{aligned}
& \varphi_{2}(x \vee y)=e^{2 i a \xi / h} e^{-\eta^{2} / 2 h} \sqrt{2 \pi h}+\mathcal{O}(1) \\
& \varphi_{1}(x \wedge y)=\mathcal{O}(1)
\end{aligned}
$$

and:

$$
\begin{aligned}
& \varphi_{2}(x \vee y)=e^{2 i a \xi / h} \mathcal{O}(1) \\
& \varphi_{1}(x \wedge y)=e^{-\eta^{2} / 2 h} \sqrt{2 \pi h}+e^{-2 i a \xi / h} \mathcal{O}(1)
\end{aligned}
$$


and we see that modulo an error $\mathcal{O}(1), K(\xi)$ is again a tensor product near $(0,0)$ and $(a, a)$. So we will tile up the $(x, y)$-plane in different domains where $K(\xi)$ is of the form $f_{j}(x) g_{j}(y)$ (possibly modulo a small term,) and label the functions $f_{j}(x), g_{j}(y)$ accordingly.

We can now give precisely the structure of $K(\xi)$. Let (again identifying an operator with its kernel):

$$
T(\xi)=\sum_{j=1}^{12} f_{j}(x) g_{j}(y)
$$

With Dirac notations, $f_{j}(x)$ will range among:

$$
\{<1 \pm|,<2-|,<5 \pm|,<6-|,<8+|,<9 \pm|,<12+|,<13 \pm|\}
$$

and $g_{j}(y)$ :

$$
\{| \pm 1\rangle,|-2\rangle,| \pm 5\rangle,|-6\rangle,|+8\rangle,| \pm 9\rangle,|+12\rangle,| \pm 13\rangle\}
$$

respectively (precise labelling will be given in (1.5),) where:

$$
\begin{aligned}
& \langle 1+|=\chi(x<\delta) e^{i x \xi / h} \varphi_{1}(x) \\
& \langle 1-|=\chi(x<\delta) e^{-i x \xi / h} \\
& \langle 2-|=\chi(x<-\delta) e^{-i x \xi / h} \\
& \langle 5+|=\chi(\delta<x<a-\delta) e^{i x \xi / h} \\
& \langle 5-|=\chi(\delta<x<a-\delta) e^{-i x \xi / h} \varphi_{2}(x) \\
& \langle 6-|=\chi(-\delta<x<\delta) e^{-i x \xi / h} e^{2 i a \xi / h} e^{-\eta^{2} / 2 h} \sqrt{2 \pi h} \\
& \langle 8+|=\chi(a-\delta<x<a+\delta) e^{i x \xi / h} e^{-\eta^{2} / 2 h} \sqrt{2 \pi h} \\
& \langle 9+|=\chi(\delta<x<a-\delta) e^{i x \xi / h} \varphi_{1}(x) \\
& \langle 9-|=\chi(\delta<x<a-\delta) e^{-i x \xi / h} \\
& \langle 12+|=\chi(x>a+\delta) e^{i x \xi / h} \\
& \langle 13+|=\chi(x>a-\delta) e^{i x \xi / h} \\
& \langle 13-|=\chi(x>a-\delta) e^{-i x \xi / h} \varphi_{2}(x) \\
& |+1\rangle=\chi(\delta<y) e^{-i y \xi / h} \psi_{+}(y) \\
& |-1\rangle=\chi(\delta<y) e^{i y \xi / h} \psi-(y) \varphi_{2}(y)
\end{aligned}
$$




$$
\begin{aligned}
& |-2\rangle=\chi(-\delta<y<\delta) e^{i y \xi / h} \psi_{-}(y) \varphi_{2}(y) \\
& |+5\rangle=\chi(y<\delta) e^{-i y \xi / h} \psi_{+}(y) \varphi_{1}(y) \\
& |-5\rangle=\chi(y<\delta) e^{i y \xi / h} \psi_{-}(y) \\
& |-6\rangle=|-5\rangle \\
& |+8\rangle=\chi(a-\delta<y) e^{-i y \xi / h} \psi_{+}(y) \\
& |+9\rangle=|+8\rangle \\
& |-9\rangle=\chi(a-\delta<y) e^{i y \xi / h} \psi_{-}(y) \varphi_{2}(y) \\
& |+12\rangle=\chi(a-\delta<y<a+\delta) e^{-i y \xi / h} \psi_{+}(y) \varphi_{1}(y) \\
& |+13\rangle=\chi(y<a-\delta) e^{-i y \xi / h} \psi_{+}(y) \varphi_{1}(y) . \\
& |-13\rangle=\chi(y<a-\delta) e^{i y \xi / h} \psi_{-}(y) .
\end{aligned}
$$

Here we have put $\delta=h^{1 / s}$ as above, and omitted the subscript $\theta$ in the formulae, according to our abuse of notation. Note that we could have probably "cut-off the tails" of the $g_{j}(y)$ 's for $y$ outside $[-\delta, \delta]$ and $[a-\delta, a+\delta]$, but then new remainder terms had to be estimated. Actually the resonant functions will be built (in a good approximation) from a linear combination of the $f_{j}$ 's. Now we define the remainder term. We have:

$$
K(\xi)=T(\xi)+H^{\prime}(\xi)
$$

In Appendix we sketch a proof of the following:

Lemma 1.1. Let $s>3, C_{0}>0$ and $\eta \in \mathbf{C}$ as above such that $|\eta| \leq C_{0} h^{1-1 / s}$, $\operatorname{Im} \eta \leq 0$. Then for any $b>0$, there exists $\theta_{b}>0$ such that if $0<\theta<\theta_{b}$ we have:

$$
H^{\prime}(\xi)=\mathcal{O}(1)
$$

in operator norm $L^{2}\left(\Gamma_{\theta}\right) \rightarrow L^{2}\left(\Gamma_{\theta}\right)$, when $h>0$ is sufficiently small.

The estimates on $K^{\prime}(\xi)$ are not so good; this is why we need shrink from below the domain where we are looking for resonances. In Appendix we sketch a proof of:

Lemma 1.2. Under the same hypotheses as in Lemma 1.1, for all $b>0$, there exists $\theta_{b}>0$ such that if $\theta<\theta_{b}$ and $b^{\prime}>b$ : 


$$
K^{\prime}(\xi)=\mathcal{O}\left(e^{-\left(a+b^{\prime}\right) \operatorname{Im} \eta / 2 h}\right): L^{2}\left(\Gamma_{\theta}\right) \rightarrow L^{2}\left(\Gamma_{\theta}\right)
$$

for $h>0$ small enough.

In the sequel, we shall keep the notation $b$ instead of $b^{\prime}$. We set:

$$
H(\xi)=K^{\prime}(\xi)+H^{\prime}(\xi)
$$

It follows from Lemmas 1.1 and 1.2 that for given $b$ and $\theta$ as above, and any $\varepsilon>0$, we have $\|\gamma(\xi) H(\xi)\|<1$ when $-\frac{4-\varepsilon}{a+b} h^{1-1 / s}<\operatorname{Im} \eta$, and $h>0$ is small enough. Next we estimate the $L^{2}$-norm of the $f_{j}$ 's and $g_{i}$ 's; along the same lines as in Lemma 1.1 we easily show:

Lemma 1.3. Under the same hypotheses as in Lemma 1.1, we have:

$$
\begin{array}{rlrl}
\langle 1+| & =\mathcal{O}(1) & & |+1\rangle=e^{-a \operatorname{Im} \eta / 2 h} \mathcal{O}(1) \\
\langle 1-|=e^{-b \operatorname{Im} \eta / 2 h} \mathcal{O}(1) & |-1\rangle=e^{-a \operatorname{Im} \eta / 2 h} \mathcal{O}(1) \\
\langle 2-|=e^{-b \operatorname{Im} \eta / 2 h} \mathcal{O}(1) & |-2\rangle=e^{-a \operatorname{Im} \eta / h} \mathcal{O}(1) \\
\langle 5+|=e^{-a \operatorname{Im} \eta / 2 h} \mathcal{O}(1) & |+5\rangle=\mathcal{O}(1) \\
\langle 5-|=e^{-a \operatorname{Im} \eta / h} \mathcal{O}(1) & |-5\rangle=\mathcal{O}(1) \\
\langle 6-|=e^{-a \operatorname{Im} \eta / h} \mathcal{O}(1) & |-6\rangle=\mathcal{O}(1) \\
\langle 8+|=e^{-a \operatorname{Im} \eta / 2 h} \mathcal{O}(1) & |+8\rangle=e^{-a \operatorname{Im} \eta / 2 h} \mathcal{O}(1) \\
\langle 9+|=e^{-a \operatorname{Im} \eta / 2 h} \mathcal{O}(1) & |+9\rangle=e^{-a \operatorname{Im} \eta / 2 h} \mathcal{O}(1) \\
\langle 9-|=\mathcal{O}(1) & |-9\rangle=e^{-a \operatorname{Im} \eta / 2 h} \mathcal{O}(1) \\
\langle 12+|=e^{-(a+b) \operatorname{Im} \eta / 2 h} \mathcal{O}(1) & |+12\rangle=e^{-a \operatorname{Im} \eta / 2 h} \mathcal{O}(1) \\
\langle 13+|=e^{-(a+b) \operatorname{Im} \eta / 2 h} \mathcal{O}(1) & |+13\rangle=\mathcal{O}(1) \\
\langle 13-|=e^{-a \operatorname{Im} \eta / 2 h} \mathcal{O}(1) & |-13\rangle=\mathcal{O}(1)
\end{array}
$$

where all the estimates are understood as $L^{2}\left(\Gamma_{\theta}\right)$ norms.

To simplify the notations we have disregarded possible powers of $h$ as prefactors for they are irrelevant. Now we are ready to compute the inverse for $I-(G(\xi) V)^{2}$. Let $f_{j}^{\prime}=(I-\gamma(\xi) H(\xi))^{-1} f_{j}$ and $T^{\prime}(\xi)$ the operator with kernel $T^{\prime}(\xi)=\sum_{j=1}^{12} f_{j}^{\prime}(x) g_{j}(y)$. We have:

$$
I-(G(\xi) V)^{2}=(I-\gamma(\xi) H(\xi))\left(I-\gamma(\xi) T^{\prime}(\xi)\right)
$$

When $-\operatorname{Im} \eta$ is not too large as above, the operator $(I-\gamma(\xi) H(\xi))^{-1}$ makes 
sense as a Neumann series. The inversion of $I-\gamma(\xi) T^{\prime}(\xi)$ is standard; namely, to solve the equation $\left(I-\gamma(\xi) T^{\prime}(\xi)\right) u=v$, we try $u=v+\gamma(\xi) \sum_{j=1}^{12} X_{j} f_{j^{\prime}}^{\prime}$, and set $Y_{j}=\left\langle v, g_{j}\right\rangle$. Using that the $f_{j}^{\prime \prime}$ s are linearly independent, we are led to the system:

$$
X_{i}-\sum_{j=1}^{12} c_{i j}(\xi) X_{j}=Y_{i}, \quad i=1,2, \cdots, 12
$$

where $c_{i j}(\xi)=\gamma(\xi)\left\langle f_{j}^{\prime} \mid g_{i}\right\rangle$.

\section{§2. The Interaction Matrix}

Before we proceed we need to recall some definitions; in the sequel we shall compute many wave packet interferences, that is, gaussian integrals. We recall from [Sj1] the precise definition of classical analytic symbols, like $a(x, h)=\Sigma_{k \geq 0} a_{k}(x) h^{k}$. A realization can be obtained by summing up the first $\sim h^{-1}$ terms, and we shall denote it again by $a(x, h)$. Classical analytic symbols in $h^{\prime}$ introduced in [Le] occur also in the treatment of Gevrey singularities. They are of the form : $a\left(x, h, h^{\prime}\right)=\Sigma_{k \geq 0} a_{k}(x, h) h^{\prime k}$ and a realization is obtained by summing up the first $\sim h^{\prime-1}$ terms. Since $h^{k}=\left(h^{2 k / s}\right) h^{\prime k}$, classical analytic symbols in $h$ are, a fortiori, classical analytic symbols in $h^{\prime}$. Now we are ready to compute the scalar products $\left\langle f_{j}^{\prime} \mid g_{i}\right\rangle$, and start with the leading term $\left\langle f_{j} \mid g_{i}\right\rangle$. We set $\kappa(\xi)=\sqrt{2 \pi h} e^{-\eta^{2} / 2 h} e^{2 i a \xi / h}$.

Lemma 1.4. Under the same conditions as in the previous Lemmas, we have:

$$
\begin{gathered}
\langle 1+\mid+5\rangle=\langle 1+\mid+13\rangle=\mathcal{O}\left(h^{3 / 2}\right) \\
\langle 1-\mid-2\rangle=\sqrt{2 \pi h} e^{-\eta^{2} / 2 h} \kappa(\xi)+\mathcal{O}(h) \\
\langle 1-\mid-5\rangle=\langle 1-\mid-6\rangle=\langle 1-\mid-13\rangle=\sqrt{2 \pi h} e^{-\eta^{2} / 2 h}+\mathcal{O}\left(e^{-1 / C h^{\prime}}\right) \\
\langle 6-\mid-2\rangle=\kappa(\xi)\left(2 \pi h e^{-\eta^{2} / h} e^{2 i a \xi / h}+\mathcal{O}(h)\right) \\
\langle 6-\mid-5\rangle=\langle 6-\mid-6\rangle=\langle 6-\mid-13\rangle=\sqrt{2 \pi h} e^{-\eta^{2} / 2 h} \kappa(\xi)+\mathcal{O}\left(e^{-1 / C h^{\prime}}\right) \\
\langle 8+\mid+1\rangle=\langle 8+\mid+8\rangle=\langle 8+\mid+9\rangle=\sqrt{2 \pi h} e^{-\eta^{2} / 2 h} \kappa(\xi)+\mathcal{O}\left(e^{-1 / C h^{\prime}}\right) \\
\langle 8+\mid+12\rangle=\sqrt{2 \pi h} e^{-\eta^{2} / 2 h}\left(\sqrt{2 \pi h} e^{-\eta^{2} / 2 h} \kappa(\xi)+\mathcal{O}(h)\right) \\
\langle 13+\mid+1\rangle=\langle 13+\mid+8\rangle=\langle 13+\mid+9\rangle=\kappa(\xi)+\mathcal{O}\left(e^{-1 / C h^{\prime}}\right)
\end{gathered}
$$




$$
\begin{aligned}
& \langle 13+\mid+12\rangle=\sqrt{2 \pi h} e^{-\eta^{2} / 2 h} \kappa(\xi)+\mathcal{O}(h) \\
& \langle 13-\mid-1\rangle=\langle 13-\mid-9\rangle=\mathcal{O}\left(h^{3 / 2}\right) e^{2 i a \xi / h}
\end{aligned}
$$

while all other brackets entering the definition of $T(\xi)$ are either 0 (for reason of support $)$, or $\mathcal{O}\left(e^{-1 / C h^{\prime}}\right)$.

Sketch of the proof. Most of brackets are obtained using (1.2), Lemma a.1, and (non-) stationary phase arguments. In particular,

$$
\begin{aligned}
& \alpha\left(x, \eta^{\prime}\right)=\mathcal{O}\left(e^{-1 / C h^{\prime}}\right), \quad|x|>\delta \\
& \alpha(x, \eta)=\sqrt{2 \pi h} e^{-\eta^{2} / 2 h}+\mathcal{O}\left(e^{-1 / C h^{\prime}}\right), \quad x>\delta \\
& \alpha(x, \eta)=\mathcal{O}\left(e^{-1 / C h^{\prime}}\right), \quad x<-\delta
\end{aligned}
$$

We are left with scalar products such as $\langle 1+\mid+5\rangle$ or $\langle 13-\mid-1\rangle$, whose leading terms are given by $I_{m}=\int_{-\delta}^{\delta} e^{-y^{2} / 2 h} e^{i y \eta / h} \alpha^{m}(y, \eta) d y, \quad m=1,2$,

$$
\text { or } \quad I_{m, n}=\int_{-\delta}^{\delta} e^{-y^{2} / 2 h} e^{i y \eta / h} \alpha^{m}(y, \eta) \alpha^{n}(-y, \eta) d y, \quad m+n=1,2
$$

But $I_{1}=e^{-\eta^{2} / 2 h} \int e^{-y^{2} / 2 h} \alpha(y-i \eta, \eta) d y+\mathcal{O}\left(e^{-1 / C h^{\prime}}\right)$ and analytic stationary phase gives

$$
e^{\eta^{2} / 2 h}(2 \pi h)^{-1 / 2} I_{1}=\alpha(-i \eta, \eta)+\mathcal{O}(\eta)
$$

while $\alpha(-i \eta, \eta)=e^{-\eta^{2} / 2 h}\left(\frac{1}{2}(2 \pi h)^{1 / 2}+\mathcal{O}(\eta)\right)$. The integrals $I_{2}$ or $I_{m, n}$ are computed similarly.

Q.E.D.

Remark. Actually, the $I_{m}$ 's or $I_{m . n}$ 's are classical analytic symbols in $h^{\prime}$, as well as all the terms $\mathcal{O}(h)$ or $\mathcal{O}\left(h^{3 / 2}\right)$ in the Lemma.

We now estimate the remainder terms $\left\langle f_{j}^{\prime} \mid g_{i}\right\rangle$, i.e. $\left\langle\left(\gamma(\xi) H(\xi)+\gamma^{2}(\xi) H^{2}(\xi)+\cdots\right) f_{j} \mid g_{i}\right\rangle$. It is clear that the rough $L^{2}$ estimate from Lemmas 1.1 to 1.3 are not sufficient, since some $f_{j}$ or $g_{i}$ are of temperate growth in the scales $\exp c / h^{1 / s}$. Nevertheless, it suffices to estimate the term which contains the first power of $H(\xi)$, for the higher order terms $\left\langle\left(\gamma^{2}(\xi) H^{2}(\xi)+\cdots\right) f_{j} \mid g_{i}\right\rangle$ can be controlled only by the $L^{2}$ norms. This amounts 
to saying that $H(\xi)$ acts only once in perturbation. We shall denote by $a(x, h)=a(x, \xi, h)$ the realization of analytic symbols. Further we adopt the following conventions: let $v_{j} \in\{0, \pm 1\}, k_{j} \in\{1,2,3, \cdots\}, l_{j} \in\{0, \pm 1, \pm 2, \cdots\}$, $m_{j} \in\{0,1,2\}, n_{j} \in\{0,1,2\}$, be some finite sequences of integers. We denote by $I_{+}$(resp. $I_{-}$) some finite subset of $\mathbf{N}$ such that $l_{j}$ is even (resp. odd) for $j \in I_{+}$(resp. $j \in I_{-}$), and $m_{j}+n_{j} \leq 2 . \quad$ Let $S_{+}(x)=\sum_{j \in I_{+}} e^{-k_{j} x^{2} / 2 h} e^{i x\left(v_{j} \eta+l_{j}\right) / h} \alpha^{m_{j}}(x, \eta)$ $\alpha^{n}(-x, \eta) a_{j}(x, h) ; S_{-}(x)$ is defined by the same formula with $I_{+}$replaced by $I_{-}$. If $I$ is a finite subset of $\mathbb{N}$, we write:

$$
T(u, v)=\sum_{j \in I} \int_{u}^{v} e^{-k_{j} y^{2} / 2 h} e^{i y v_{j} \eta / h} \alpha^{m_{j}}(y, \eta) \alpha^{n_{j}}(-y, \eta) a_{j}(y, h) d y
$$

As usual, by $S_{ \pm}(x) \otimes T(u, v)$ we denote any finite sum of products of a term of type $S_{ \pm}(x)$ with a term of type $T(u, v)$. We use Lemma a.2. Computation of $<K^{\prime}(\xi)|j|$ involves terms like $S_{ \pm}(x) \otimes T(x, \pm \delta)$ or $S_{ \pm}(x-a) \otimes T(x-a, \pm \delta)$ with $m_{j}+n_{j}=0,1$ in the definition above. Then it remains to evaluate the other components of $H(\xi)$. Most significant terms are $\left\langle H_{6}(\xi)|j|\right.$ and $\left\langle H_{8}(\xi)|j|\right.$. Their computation is analogous to that of $\left\langle K^{\prime}(\xi)|j|\right.$, but we need also take $m_{j}+n_{j}=2$. All integrals with non stationnary phases are treated with help of Lemma a.3 and Remark a.4. As a result we get the following:

Lemma 1.5. Under the same conditions as above, with the notations just defined and omitting the subscript $\theta$, we have:

$$
\begin{aligned}
& \left\langle H(\xi)|j|=\mathcal{O}\left(e^{-1 / C h^{\prime}}\right), \quad j=2-, 5 \pm, 9 \pm, 12+\right. \\
& \left\langle H(\xi)|1+|=\chi(-\delta<x<\delta) e^{i x \xi / h}\right. \\
& \quad\left(T(-\delta, x)+S_{+}(x)+\chi(x, \eta) T(x, \delta)+S_{+}(x) \otimes T(x, \delta)\right) \\
& \quad+\chi(-\delta<x<\delta) e^{-i x \xi / h}\left(S_{-}(x) \otimes T(x, \delta)+S_{-}(x)\right) \\
& \quad+\chi(x>\delta) e^{i x \xi / h} \mathcal{O}(1)+\mathcal{O}\left(e^{-1 / C h^{\prime}}\right) \\
& \quad H(\xi)|1-|=\chi(-\delta<x<\delta) e^{i x \xi / h} S_{-}(x) \\
& \quad+\chi(x<\delta) e^{-i x \xi / h}\left(T(x, \delta)+\alpha(-x, \eta) \alpha(x, \eta)+S_{+}(x)\right) \\
& \quad+e^{-\eta^{2} / 2 h} \sqrt{2 \pi h} \chi(a-\delta<x<a+\delta) e^{i x \xi / h} S_{-}(x-a)+\mathcal{O}\left(e^{-1 / C h^{\prime}}\right) \\
& <H(\xi)|6-|=\kappa(\xi) \chi(-\delta<x<\delta) e^{i x \xi / h} S_{-}(x) \\
& \quad+\kappa(\xi) \chi(-\delta<x<\delta) e^{-i x \xi / h}\left(T(x, \delta)+T(-\delta, x)+S_{+}(x)\right)
\end{aligned}
$$$$
3^{\circ}
$$$$
4^{\circ}
$$ 


$$
\begin{aligned}
& +\kappa(\xi) \chi(x<-\delta) e^{-i x \xi / h} \mathcal{O}(1) \\
& +\kappa(\xi) \chi(a-\delta<x<a+\delta) e^{i x \xi / h} S_{-}(x-a)+\mathcal{O}\left(e^{-1 / C h^{\prime}}\right) .
\end{aligned}
$$

$5^{\circ}$

$$
\begin{aligned}
& \left\langle H(\xi)|8+|=\sqrt{2 \pi h} e^{-\eta^{2} / 2 h} \kappa(\xi) \chi(-\delta<x<\delta) e^{-i x \xi / h} S_{-}(x)\right. \\
& \quad+\sqrt{2 \pi h} e^{-\eta^{2} / 2 h} \chi(a-\delta<x<a+\delta) e^{i x \xi / h} \\
& \left(T(-\delta, x-a)+\alpha(x-a, \eta) \alpha(a-x, \eta) T(-\delta, x-a)+S_{+}(x-a)\right) \\
& \quad+\kappa(\xi) \chi(a-\delta<x<a+\delta) e^{-i x \xi / h} S_{-}(x-a) \\
& \quad+\sqrt{2 \pi h} e^{-\eta^{2} / 2 h} \chi(x>a+\delta) e^{i x \xi / h} \mathcal{O}(1)+\mathcal{O}\left(e^{-1 / C h^{\prime}}\right) .
\end{aligned}
$$

$6^{\circ}\left\langle H(\xi)|13+|=\kappa(\xi) \chi(x<a-\delta) e^{-i x \xi / h} S_{-}(x)\right.$

$$
\begin{aligned}
& +\chi(a-\delta<x<a+\delta) e^{i x \xi / h}\left(T(-\delta, x-a)+\alpha(x-a, \eta) \alpha(a-x, \eta)+S_{+}(x-a)\right) \\
& +e^{2 i a \xi / h} \chi(a-\delta<x<a+\delta) e^{-i x \xi / h} S_{-}(x-a) \\
& +\chi(x>a+\delta) e^{i x \xi / h} \mathcal{O}(1)+\mathcal{O}\left(e^{-1 / C h^{\prime}}\right)
\end{aligned}
$$

$7^{\circ}\left\langle H(\xi)|13-|=\chi(a-\delta<x<a+\delta) e^{i x \xi / h}\left(S_{-}(x-a) \otimes T(-\delta, x-a)+S_{-}(x-a)\right)\right.$

$$
\begin{aligned}
& +e^{2 i a \xi / h} \chi(a-\delta<x<a+\delta) e^{-i x \xi / h} \\
& \left(T(x-a, \delta)+\alpha(a-x, \eta) T(-\delta, x-a)+S_{+}(x-a) \otimes T(-\delta, x-a)+S_{+}(x-a)\right) \\
& +e^{2 i a \xi / h} \chi(x<a-\delta) e^{-i x \xi / h} \mathcal{O}(1)+\mathcal{O}\left(e^{-1 / C h^{\prime}}\right) .
\end{aligned}
$$

Here all estimates $\mathcal{O}\left(e^{-1 / C h^{\prime}}\right)$ are uniform in $L^{2}\left(\Gamma_{\theta}\right)$ norm and $\eta \in \mathbf{C},|\eta|<C_{0} h^{1-1 / s}$, $\operatorname{Im} \eta \leq 0$, if $h>0$ is small enough; the $\mathcal{O}(1)$ terms are constant, just depending on $h$ and $\xi$.

Now we can compute the matrix elements $\left\langle f_{j}^{\prime} \mid g_{i}\right\rangle, 1 \leq i, j \leq 12$. If quantities $\left\langle\gamma(\xi) H(\xi)\left|f_{j}\right| g_{i}\right\rangle$ are evaluated with the same accuracy as in Lemma 1.4, then the remainder term $\left\langle\left(\gamma^{2}(\xi) H^{2}(\xi)+\cdots\right)\left|f_{j}\right| g_{i}\right\rangle$ can be estimated with a crude $L^{2}$-inequality. So the accuracy to which matrix elements are computed in the following Lemma depends on the terms. This remarkable fact hinges on the structure of the matrix which will be discussed below. Now we state precisely (and with a comfortable margin) the domain allowed for $\eta$. For $b>0$ small enough, we put:

$$
W_{h}=\left\{\eta \in \mathbf{C}: \operatorname{Im} \eta \leq 0,|\eta|<C_{0} h^{1-1 / s}, a \operatorname{Im} \eta+2 h^{1-1 / s}>-\frac{b}{a} h^{1-1 / s}\right\}
$$


We set also $\left\langle j^{\prime} \pm\right|=\left\langle(I-\gamma(\xi) H(\xi))^{-1}|j \pm|\right.$. After very tedious but straightforward computations, we have as in Lemma 1.4:

Lemma 1.6. For $\eta \in W_{h}$, the following estimates hold when $h>0$ is small enough:

$$
\begin{aligned}
& 1^{\circ}\left\langle 1^{\prime}+\mid+1\right\rangle=\gamma(\xi) e^{2 i a \xi / h} \mathcal{O}(1) \\
& \left\langle 1^{\prime}+\mid-1\right\rangle=e^{-h^{-1 / s}} e^{6 b / a h^{1 / s}} \mathcal{O}(1) \\
& \left\langle 1^{\prime}+\mid-2\right\rangle=e^{6 b / a h^{1 / s}} \mathcal{O}(1) \\
& \left\langle 1^{\prime}+\mid+5\right\rangle=\mathcal{O}(1) \\
& \left\langle 1^{\prime}+\mid-5\right\rangle=\left\langle 1^{\prime}+\mid-6\right\rangle=e^{-2 h^{-1 / s}} e^{6 b / a h^{1 / s}} \mathcal{O}(1) \\
& \left\langle 1^{\prime}+\mid+8\right\rangle=\left\langle 1^{\prime}+\mid+9\right\rangle=\gamma(\xi) e^{2 i a \xi / h} \mathcal{O}(1) \\
& \left\langle 1^{\prime}+\mid-9\right\rangle=e^{-h^{-1 / s}} e^{6 b / a h^{1 / s}} \mathcal{O}(1) \\
& \left\langle 1^{\prime}+\mid+12\right\rangle=\gamma(\xi) e^{2 i a \xi / h} \mathcal{O}(1) \\
& \left\langle 1^{\prime}+\mid+13\right\rangle=\mathcal{O}(1) \\
& \left\langle 1^{\prime}+\mid-13\right\rangle=e^{-2 h^{-1 / s}} e^{6 b / a h^{1 / s}} \mathcal{O}(1) \\
& 2^{\circ}\left\langle 1^{\prime}-\mid+1\right\rangle=e^{-h^{-1 / s}} e^{6 b / a h^{1 / s}} \mathcal{O}(1) \\
& \left\langle 1^{\prime}-\mid-1\right\rangle=\gamma(\xi) e^{2 i a \xi / h} \mathcal{O}(1) \\
& \left\langle 1^{\prime}-\mid-2\right\rangle=\sqrt{2 \pi h} e^{-\eta^{2} / 2 h} \kappa(\xi)\left(1+e^{-2 h^{-1 / s}} e^{6 b / a h^{1 / s}} \mathcal{O}(1)\right) \\
& \left\langle 1^{\prime}-\mid+5\right\rangle=e^{-2 h^{-1 / s}} e^{6 b / a h^{1 / s}} \mathcal{O}(1) \\
& \left\langle 1^{\prime}-\mid-5\right\rangle=\left\langle 1^{\prime}-\mid-6\right\rangle=\sqrt{2 \pi h} e^{-\eta^{2} / 2 h}\left(1+e^{-2 h^{-1 / s}} e^{6 b / a h^{1 / s}} \mathcal{O}(1)\right) \\
& \left\langle 1^{\prime}-\mid+8\right\rangle=\left\langle 1^{\prime}-\mid+9\right\rangle=e^{-h^{-1 / s}} e^{6 b / a h^{1 / s}} \mathcal{O}(1) \\
& \left\langle 1^{\prime}-\mid-9\right\rangle=\gamma(\xi) e^{2 i a \xi / h} \mathcal{O}(1) \\
& \left\langle 1^{\prime}-\mid+12\right\rangle=e^{-h^{-1 / s}} e^{6 b / a h^{1 / s}} \mathcal{O}(1) \\
& \left\langle 1^{\prime}-\mid+13\right\rangle=e^{-2 h^{-1 / s}} e^{6 b / a h^{1 / s}} \mathcal{O}(1) \\
& \left\langle 1^{\prime}-\mid-13\right\rangle=\sqrt{2 \pi h} e^{-\eta^{2} / 2 h}\left(1+e^{-2 h^{-1 / s}} e^{6 b / a h^{1 / s}} \mathcal{O}(1)\right) \\
& 3^{\circ}\left\langle 6^{\prime}-\mid+1\right\rangle=e^{h^{-1 / s}} e^{6 b / a h^{1 / s}} \mathcal{O}(1) \\
& \left\langle 6^{\prime}-\mid-1\right\rangle=\gamma(\xi) e^{4 i a \xi / h} \mathcal{O}(1)
\end{aligned}
$$




$$
\begin{aligned}
& \left\langle 6^{\prime}-\mid-2\right\rangle=2 \pi h e^{-\eta^{2} / h} \kappa(\xi) e^{2 i a \xi / h}\left(1+e^{-2 h^{-1 / s}} e^{6 b / a h^{1 / s}} \mathcal{O}(1)\right) \\
& \left\langle 6^{\prime}-\mid+5\right\rangle=e^{6 b / a h^{1 / s}} \mathcal{O}(1) \\
& \left\langle 6^{\prime}-\mid-5\right\rangle=\left\langle 6^{\prime}-\mid-6\right\rangle=\sqrt{2 \pi h} e^{-\eta^{2} / 2 h} \kappa(\xi)\left(1+e^{-2 h^{-1 / s}} e^{6 b / a h^{1 / s}} \mathcal{O}(1)\right) \\
& \left\langle 6^{\prime}-\mid+8\right\rangle=\left\langle 6^{\prime}-\mid+9\right\rangle=e^{h^{-1 / s}} e^{6 b / a h^{1 / s}} \mathcal{O}(1) \\
& \left\langle 6^{\prime}-\mid-9\right\rangle=\gamma(\xi) e^{4 i a \xi / h} \mathcal{O}(1) \\
& \left\langle 6^{\prime}-\mid+12\right\rangle=e^{h^{-1 / s}} e^{6 b / a h^{1 / s}} \mathscr{O}(1) \\
& \left\langle 6^{\prime}-\mid+13\right\rangle=e^{6 b / a h^{1 / s}} \mathcal{O}(1) \\
& \left\langle 6^{\prime}-\mid-13\right\rangle=\sqrt{2 \pi h} e^{-\eta^{2} / 2 h} \kappa(\xi)\left(1+e^{-2 h^{-1 / s}} e^{6 b / a h^{1 / s}} \mathcal{O}(1)\right) \\
& 4^{\circ}\left\langle 8^{\prime}+\mid+1\right\rangle=\sqrt{2 \pi h} e^{-\eta^{2} / 2 h} \kappa(\xi)\left(1+e^{-2 h^{-1 / s}} e^{6 b / a h^{1 / s}} \mathcal{O}(1)\right) \\
& \left\langle 8^{\prime}+\mid-1\right\rangle=e^{6 b / a h^{1 / s}} \mathcal{O}(1) \\
& \left\langle 8^{\prime}+\mid-2\right\rangle=e^{h^{-1 / s}} e^{6 b / a h^{1 / s}} \mathcal{O}(1) \\
& \left\langle 8^{\prime}+\mid+5\right\rangle=\gamma(\xi) e^{2 i a \xi / h} \mathscr{O}(1) \\
& \left\langle 8^{\prime}+\mid-5\right\rangle=\left\langle 8^{\prime}+\mid-6\right\rangle=e^{-h^{-1 / s}} e^{6 b / a h^{1 / s}} \mathcal{O}(1) \\
& \left\langle 8^{\prime}+\mid+8\right\rangle=\left\langle 8^{\prime}+\mid+9\right\rangle=\sqrt{2 \pi h} e^{-\eta^{2} / 2 h} \kappa(\xi)\left(1+e^{-2 h^{-1 / s}} e^{6 b / a h^{1 / s}} \mathcal{O}(1)\right) \\
& \left\langle 8^{\prime}+\mid-9\right\rangle=e^{6 b / a h^{1 / s}} \mathcal{O}(1) \\
& \left\langle 8^{\prime}+\mid+12\right\rangle=2 \pi h e^{-\eta^{2} / h} \kappa(\xi)\left(1+e^{-2 h^{-1 / s}} e^{6 b / a h^{1 / s}} \mathcal{O}(1)\right) \\
& \left\langle 8^{\prime}+\mid+13\right\rangle=\gamma(\xi) e^{2 i a \xi / h} \mathcal{O}(1) \\
& \left\langle 8^{\prime}+\mid-13\right\rangle=e^{-h^{-1 / s}} e^{6 b / a h^{1 / s}} \mathcal{O}(1) \\
& 5^{\circ}\left\langle 13^{\prime}+\mid+1\right\rangle=\kappa(\xi)\left(1+e^{-2 h^{-1 / s}} e^{6 b / a h^{1 / s}} \mathcal{O}(1)\right) \\
& \left\langle 13^{\prime}+\mid-1\right\rangle=e^{6 b / a h^{1 / s}} \mathcal{O}(1) \\
& \left\langle 13^{\prime}+\mid-2\right\rangle=e^{h^{-1 / s}} e^{6 b / a h^{1 / s}} \mathcal{O}(1) \\
& \left\langle 13^{\prime}+\mid+5\right\rangle=\gamma(\xi) e^{2 i a \xi / h} \mathcal{O}(1) \\
& \left\langle 13^{\prime}+\mid-5\right\rangle=\left\langle 13^{\prime}+\mid-6\right\rangle=e^{-h^{-1 / s}} e^{6 b / a h^{1 / s}} \mathcal{O}(1) \\
& \left\langle 13^{\prime}+\mid+8\right\rangle=\left\langle 13^{\prime}+\mid+9\right\rangle=\kappa(\xi)\left(1+e^{-2 h^{-1 / s}} e^{6 b / a h^{1 / s}} \mathcal{O}(1)\right) \\
& \left\langle 13^{\prime}+\mid-9\right\rangle=e^{6 b / a h^{1 / s}} \mathfrak{C}(1) \\
& \left\langle 13^{\prime}+\mid+12\right\rangle=\sqrt{2 \pi h} e^{-\eta^{2} / 2 h} \kappa(\xi)\left(1+e^{-2 h^{-1 / s}} e^{6 b / a h^{1 / s}} \mathcal{O}(1)\right)
\end{aligned}
$$




$$
\begin{aligned}
&\left\langle 13^{\prime}+\mid+13\right\rangle=\gamma(\xi) e^{2 i a \xi / h} \mathcal{O}(1) \\
&\left\langle 13^{\prime}+\mid-13\right\rangle=e^{-h^{-1 / s}} e^{6 b / a h^{1 / s}} \mathcal{O}(1) \\
& 6^{\circ} \quad\left\langle 13^{\prime}-\mid+1\right\rangle=e^{6 b / a h^{1 / s}} \mathcal{O}(1) \\
&\left\langle 13^{\prime}-\mid-1\right\rangle=\kappa(\xi) \mathcal{O}(h) \\
&\left\langle 13^{\prime}-\mid-2\right\rangle=\gamma(\xi) e^{4 i a \xi / h} \mathcal{O}(1) \\
&\left\langle 13^{\prime}-\mid+5\right\rangle=e^{-h^{-1 / s}} e^{6 b / a h^{1 / s}} \mathcal{O}(1) \\
&\left\langle 13^{\prime}-\mid-5\right\rangle=\left\langle 13^{\prime}-\mid-6\right\rangle=\gamma(\xi) e^{2 i a \xi / h} \mathcal{O}(1) \\
&\left\langle 13^{\prime}-\mid+8\right\rangle=\left\langle 13^{\prime}-\mid+9\right\rangle=e^{6 b / a h^{1 / s}} \mathcal{O}(1) \\
&\left\langle 13^{\prime}-\mid-9\right\rangle=\kappa(\xi) \mathcal{O}(h) \\
&\left\langle 13^{\prime}-\mid+12\right\rangle=e^{6 b / a h^{1 / s}} \mathscr{O}(1) \\
&\left\langle 13^{\prime}-\mid+13\right\rangle=e^{-h^{-1 / s}} e^{6 b / a h^{1 / s}} \mathcal{O}(1) \\
&\left\langle 13^{\prime}-\mid-13\right\rangle=\gamma(\xi) e^{2 i a \xi / h} \mathcal{O}(1)
\end{aligned}
$$

At last we get the important relations (see Lemmas 1.4 and $1.5,1^{\circ}$ ):

$$
\left\langle j^{\prime} \mid \pm i\right\rangle=\mathcal{O}\left(e^{-1 / C h^{\prime}}\right), j^{\prime}=2^{\prime}-, 5^{\prime} \pm, 9^{\prime} \pm, 12^{\prime}+, \forall i
$$

We are ready now to solve system (1.3).

\section{§3. The System}

We label as follows:

$$
\begin{aligned}
& f_{1}^{\prime}=\left\langle 6^{\prime}-\right|, f_{2}^{\prime}=\left\langle 1^{\prime}+\right|, f_{3}^{\prime}=\left\langle 1^{\prime}-\right|, f_{4}^{\prime}=\left\langle 13^{\prime}+\right|, \\
& f_{5}^{\prime}=\left\langle 13^{\prime}-\right|, f_{6}^{\prime}=\left\langle 8^{\prime}+\right|, f_{7}^{\prime}=\left\langle 2^{\prime}-\right|, f_{8}^{\prime}=\left\langle 5^{\prime}+\right|, \\
& f_{9}^{\prime}=\left\langle 5^{\prime}-\right|, f_{10}^{\prime}=\left\langle 9^{\prime}+\right|, f_{11}^{\prime}=\left\langle 9^{\prime}-\right|, f_{12}^{\prime}=\left\langle 12^{\prime}+\right|
\end{aligned}
$$

and correspondingly for the $g_{i}$ 's. We solve explicitely (1.3) modulo errors of order $e^{-C / h^{1 / s}}$. Introduce

$$
\begin{array}{ll}
X^{a}=\left(X_{1}, \cdots, X_{6}\right) & X^{b}=\left(X_{7}, \cdots, X_{12}\right) \\
Y^{a}=\left(Y_{1}, \cdots, Y_{6}\right) & Y^{b}=\left(Y_{7}, \cdots, Y_{12}\right)
\end{array}
$$

With the almost orthogonality relations (1.4), system (1.3) rewrites: 


$$
X_{i}=Y_{i}+\sum_{j=1}^{6} c_{i j}(\xi) X_{j}+\mathcal{O}\left(e^{-1 / C h^{\prime}}\right)\left(X^{b}\right), \quad i=1, \cdots, 12
$$

where $\mathcal{O}\left(e^{-1 / C h^{\prime}}\right)\left(X^{b}\right)$ denotes a linear form in $X^{b}$ with $\mathcal{O}\left(e^{-1 / C h^{\prime}}\right)$ coefficients if $\eta \in W_{h}$. The determinant of the system (1.3) vanishes of nearly second order for some discrete values of $\xi$. It will be computed in the next section. We proceed to discuss the resolution of (1.3). First we solve for $\left(X_{2}, X_{4}\right)$ vs. $\left(Y_{2}, Y_{4}, X_{1}, X_{3}, X_{5}, X_{6}, X^{b}\right)$. For $\eta \in W_{h}$, the determinant of this $2 \times 2$ system is $1+\gamma^{2}(\xi) e^{2 i a \xi / h} \mathcal{C}(1)$, so is very close to 1 . Then we plug $\left(X_{2}, X_{4}\right)$ in (1.6) $)_{6}$. This gives a linear equation for $X_{6}$ vs. $\left(Y_{2}, Y_{4}, Y_{6}, X_{1}, X_{3}, X_{5}, X^{b}\right)$. The coefficient of $X_{6}$ writes:

$$
d_{6}(\xi)=1-\gamma(\xi) 2 \pi h e^{-\eta^{2} / 2 h} e^{2 i a \xi / h}\left(1+e^{-2 h^{-1 / s}} e^{6 b / a h^{1 / s}} \mathcal{O}(1)\right)
$$

The roots of $d_{6}(\xi)$ will be shown to be exponentially close (in the scales $e^{-C / h^{1 / s}}$ ) to the resonances of our operator. Assume for the moment that $\xi$ is chosen in such a way that:

$$
\left|d_{6}(\xi)\right| \geq \text { Const. }>0
$$

(this condition could be substantially weakened, e.g. $\left|d_{6}(\xi)\right| \geq e^{-C / h^{1 / s}}$ for small $C>0$, but it is sufficient to our purpose.) If we substitute $X_{6}$ vs. $\left(Y_{2}, Y_{4}, Y_{6}, X_{1}, X_{3}, X_{5}, X^{b}\right)$ into $\left(X_{2}, X_{4}\right)$ when (1.8) holds, we get $\left(X_{2}, X_{4}\right)$ vs. $\left(Y_{2}, Y_{4}, Y_{6}, X_{1}, X_{3}, X_{5}, X^{b}\right)$. Next, using (1.6) $)_{3}$ and (1.6) $)_{5}$, we solve for $\left(X_{3}, X_{5}\right)$ vs. $\left(Y_{3}, Y_{5}, X_{1}, X_{2}, X_{4}, X_{6}, X^{b}\right)$. Again, we get a $2 \times 2$ system whose determinant is $1+\gamma^{2}(\xi) e^{2 i a \xi / h} \mathcal{O}(1)$, so is very close to 1 . Then we substitute these values for $\left(X_{3}, X_{5}\right)$ in the expressions giving $\left(X_{2}, X_{4}, X_{6}\right)$; so whenever (1.8) holds, we get $\left(X_{2}, X_{4}, X_{6}\right)$ vs. $\left(Y_{2}, Y_{3}, Y_{4}, Y_{5}, Y_{6}, X_{1}, X^{b}\right)$, which in turn gives $\left(X_{3}, X_{5}\right)$ vs. $\left(Y_{2}, Y_{3}, Y_{4}, Y_{5}, Y_{6}, X_{1}, X^{b}\right)$. Substituting into (1.6) $)_{1}$ we get a linear equation for $X_{1}$ vs. $\left(Y^{a}, X^{b}\right)$, and the coefficient of $X_{1}$ is found to be:

$$
\begin{array}{r}
d_{1}(\xi)=1-\gamma(\xi) 2 \pi h e^{-\eta^{2} / h} e^{2 i a \xi / h}\left(1+e^{-2 h^{-1 / s}} e^{6 b / a h^{1 / s}} \mathcal{O}(1)\right) \\
+e^{-5 h^{-1 / s}} \frac{1}{d_{6}(\xi)} e^{6 b / a h^{1 / s}} \mathcal{O}(1)
\end{array}
$$

where $\mathcal{O}(1)$ is holomorphic in $W_{h}$; so $d_{1}(\xi)$ is very close to $d_{6}(\xi)$ on $\left|d_{6}(\xi)\right| \geq$ Const. $>0$. We still assume:

$$
\left|d_{1}(\xi)\right| \geq \text { Const. }>0
$$

Having determined $X_{1}$ vs. $\left(Y^{a}, X^{b}\right)$ we can substitute into the former expression 
giving $X^{a}$. Using also (1.4) to get rid of the $X^{b}$ dependence, we eventually obtain the following Lemma. Let us specify again some notations. By $\operatorname{Hol}(\xi)$ we mean any holomorphic function $f(\xi, h)$ of $\xi \in W_{h}$ with temperate growth in the scales $\exp c h^{1 / s}$. By $\operatorname{Mer}(\xi)$ we mean any meromorphic function $f(\xi, h)$ of $\xi \in W_{h}$ whose numerator is $e^{12 b / a h^{1 / s}} \mathcal{O}(1)$, and whose denominator is of the form $d_{1}(\xi)^{k} d_{6}(\xi)^{l}$, with $1 \leq k+l \leq 2$. We have:

Lemma 1.7. Assume $\eta \in W_{h}$ and (1.8), (1.10) hold. Then system (1.3) has a unique solution given by:

$$
\begin{aligned}
1^{\circ} & X_{1}=\frac{1}{d_{1}(\xi)} Y_{1}+e^{-4 h^{-1 / s}} \operatorname{Mer}(\xi) Y_{2} \\
& +\left(\frac{1}{d_{1}(\xi)} \sqrt{2 \pi h} e^{-\eta^{2} / 2 h} \gamma(\xi)+e^{-4 h^{-1 / s}} \operatorname{Mer}(\xi)\right) Y_{3} \\
& +e^{-3 h^{-1 / s}} \operatorname{Mer}(\xi) Y_{4}+\left(\frac{1}{d_{1}(\xi)}-\gamma^{2}(\xi) \kappa(\xi) \mathcal{O}(h)+e^{-4 h^{-1 / s}} \operatorname{Mer}(\xi)\right) Y_{5} \\
& +e^{-3 h^{-1 / s}} \operatorname{Mer}(\xi) Y_{6}+\mathcal{O}\left(e^{-1 / C h^{\prime}}\right)\left(Y^{b}\right)
\end{aligned}
$$

$2^{\circ} \quad X_{2}=e^{-h^{-1 / s}} \operatorname{Mer}(\xi) Y_{1}+\left(\operatorname{Hol}(\xi)+e^{-5 h^{-1 / s}} \operatorname{Mer}(\xi)\right) Y_{2}+e^{-3 h^{-1 / s}} \operatorname{Mer}(\xi) Y_{3}$

$+\left(\operatorname{Hol}(\xi)+\frac{1}{d_{6}(\xi)} \sqrt{2 \pi h} e^{-\eta^{2} / 2 h} \gamma^{2}(\xi) \kappa^{2}(\xi)+e^{-2 h^{-1 / s}} \operatorname{Mer}(\xi)\right) Y_{4}$

$+e^{-2 h^{-1 / s}} \operatorname{Mer}(\xi) Y_{5}+\left(\frac{1}{d_{6}(\xi)} \sqrt{2 \pi h} e^{-\eta^{2} / 2 h} \gamma(\xi) \kappa(\xi)+e^{-2 h^{-1 / s}} \operatorname{Mer}(\xi)\right) Y_{6}$

$+\mathcal{O}\left(e^{-1 / C h^{\prime}}\right)\left(Y^{b}\right)$

$3^{\circ} \quad X_{3}=\left(\frac{1}{d_{1}(\xi)} \gamma^{2}(\xi) \kappa^{2}(\xi) \mathcal{O}(1)+e^{-4 h^{-1 / s}} \operatorname{Mer}(\xi)\right) Y_{1}$

$$
+\left(\operatorname{Hol}(\xi)+e^{-4 h^{-1 / s}} \operatorname{Mer}(\xi)\right) Y_{2}+\left(\operatorname{Hol}(\xi)+e^{-2 h^{-1 / s}} \operatorname{Mer}(\xi)\right) Y_{3}
$$$$
+e^{-2 h^{-1 / s}} \operatorname{Mer}(\xi) Y_{4}+\left(\operatorname{Hol}(\xi)+e^{-2 h^{-1 / s}} \operatorname{Mer}(\xi)\right) Y_{5}
$$$$
+e^{-2 h^{-1 / s}} \operatorname{Mer}(\xi) Y_{6}+\mathcal{O}\left(e^{-1 / C h^{\prime}}\right)\left(Y^{b}\right) \text {. }
$$

$4^{\circ} \quad X_{4}=e^{-2 h^{-1 / s}} \operatorname{Mer}(\xi) Y_{1}+\left(\gamma(\xi) \mathcal{O}(1)+e^{-4 h^{-1 / s}} \operatorname{Mer}(\xi)\right) Y_{2}$

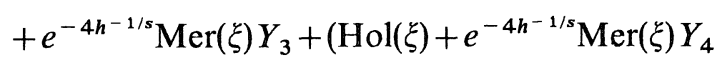

$+\left(\operatorname{Hol}(\xi)+e^{-4 h^{-1 / s}} \operatorname{Mer}(\xi)\right) Y_{5}+\left(\frac{1}{d_{6}(\xi)} \gamma^{2}(\xi) \kappa(\xi) \mathcal{O}(1)+e^{-5 h^{-1 / s}} \operatorname{Mer}(\xi)\right) Y_{6}$ 


$$
\begin{aligned}
& +\mathcal{O}\left(e^{-1 / C h^{\prime}}\right)\left(Y^{b}\right) \text {. } \\
& 5^{\circ} \quad X_{5}=\left(\frac{1}{d_{1}(\xi)} \sqrt{2 \pi h} e^{-\eta^{2} / 2 h_{\gamma}} \gamma(\xi) \kappa(\xi)+e^{-2 h-1 / s} \operatorname{Mer}(\xi)\right) Y_{1}
\end{aligned}
$$

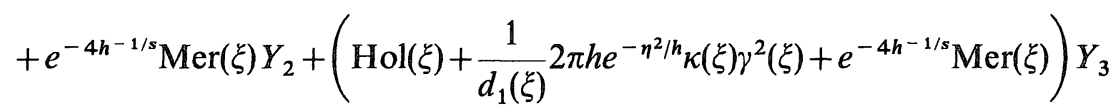

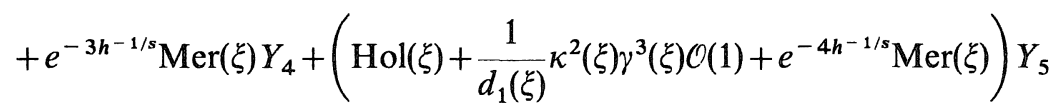

$$
\begin{aligned}
& +e^{-3 h^{-1 / s}} \operatorname{Mer}(\xi) Y_{6}+\mathcal{O}\left(e^{-1 / C h^{\prime}}\right)\left(Y^{b}\right) \text {. } \\
& 6^{\circ} \quad X_{6}=e^{-h^{-1 / s}} \operatorname{Mer}(\xi) Y_{1}+\left(\frac{1}{d_{6}(\xi)} \kappa(\xi) \gamma^{2}(\xi) \mathcal{O}(1)+e^{-5 h^{-1 / s}} \operatorname{Mer}(\xi)\right) Y_{2} \\
& +e^{-3 h^{-1 / s}} \operatorname{Mer}(\xi) Y_{3}+\left(\frac{1}{d_{6}(\xi)} \kappa(\xi) \gamma(\xi)+e^{-2 h^{-1 / s}} \operatorname{Mer}(\xi)\right) Y_{4} \\
& +e^{-h^{-1 / s}} \operatorname{Mer}(\xi) Y_{5}+\left(\frac{1}{d_{6}(\xi)}+e^{-4 h^{-1 / s}} \operatorname{Mer}(\xi)\right) Y_{6}+\mathcal{O}\left(e^{-1 / C h^{\prime}}\right)\left(Y^{b}\right)
\end{aligned}
$$

\section{§4. The Determinants}

A straightforward computation shows that the determinant of the whole system (1.3) is given by:

$$
\begin{aligned}
d_{0}(\xi)= & \left(1-2 \pi h e^{-\eta^{2} / h} e^{2 i a \xi / h} \gamma(\xi)+\mathcal{O}\left(e^{-1 / C h^{1 / s}}\right)\right) \\
& \left(1-2 \pi h e^{-\eta^{2} / h} e^{2 i a \xi / h} \gamma(\xi)+\mathcal{O}\left(e^{-1 / C h^{1 / s}}\right)\right)\left(1+\mathcal{O}\left(e^{-1 / C h^{1 / s}}\right)\right) \\
& +\mathcal{O}\left(e^{-1 / C h^{1 / s}}\right), \quad C>0 .
\end{aligned}
$$

all $\mathcal{O}$ 's being holomorphic in $W_{h}$. Now we investigate the roots of $d_{6}(\xi), d_{1}(\xi)$ and $d_{0}(\xi)$. First we rescale $\eta$ by setting $\eta=\tilde{\eta} h^{1-1 / s}$, so that $\tilde{\eta}=\mathcal{O}(1)$. For $k \in \mathbb{Z}$ such that:

$$
\frac{2 \pi}{a}|k| h<\frac{1}{2} C_{0} h^{1-1 / s}
$$

we set $\tilde{\eta}_{k}=2 k \pi h^{1 / s} / a$, and $\tilde{\eta}=\tilde{\eta}_{k}-\frac{2 i}{a}+\zeta$, where $|\operatorname{Re} \zeta|<\frac{\pi}{a} h^{1 / s},|\operatorname{Im} \zeta|<\frac{b}{a^{2}}$. Let: $B_{k}^{\prime}(h)=\left\{\eta=h^{1-1 / s}\left(\tilde{\eta}_{k}-\frac{2 i}{a}+\zeta\right),|\operatorname{Re} \zeta|<\frac{\pi}{a} h^{1 / s},|\operatorname{Im} \zeta|<\frac{b}{a^{2}}\right\}$, so that the union over 
$k \in \mathbf{Z}$ satisfying (1.12) of small neighborhoods of the $B_{k}^{\prime}(h)$ cover the "bottom" of $W_{h}$. It is clear that $d_{6}(\xi), d_{1}(\xi)$ and $d_{0}(\xi)$ do not vanish in $W_{h} \backslash \cup B_{k}^{\prime}(h)$. Next we fix $a=a(h)=(2 m+1) \pi h$ where $m \in \mathbb{N}$ is so large that $\left|a-a_{0}\right|<$ const. $h$, for some constant $a_{0}$. This is a coherence condition to get a standing wave pattern. We set:

$$
d(\xi)=1-2 \pi h e^{-\eta^{2} / h} e^{2 i a \xi / h} \gamma(\xi)
$$

We have:

$$
1-d(\xi)=\left(1+h^{1-1 / s}\left(\tilde{\eta}_{k}-\frac{2 i}{a}+\zeta\right)\right)^{-2} \exp \left[i a \zeta / h^{1 / s}\right] \exp \left[-\left(\tilde{\eta}_{k}-\frac{2 i}{a}+\zeta\right)^{2} h^{\prime}\right]
$$

We rescale again $\zeta$ by setting $\zeta=h^{1 / s} \tilde{\zeta}, \tilde{\zeta}=\mathcal{O}(1)$, which amounts to shrink $\zeta$ in $B_{k}^{\prime}(h)$; again it is clear that none of $d_{6}(\xi), d_{1}(\xi), d_{0}(\xi)$, or $d(\xi)$ vanish in $B_{k}^{\prime}(h) \backslash B_{k}(h)$, where:

$$
B_{k}(h)=\left\{\eta=h^{1-1 / s}\left(\tilde{\eta}_{k}-\frac{2 i}{a}\right)+h \tilde{\zeta},|\operatorname{Re} \tilde{\zeta}|<\frac{\pi}{a},|\operatorname{Im} \tilde{\zeta}|<C_{1}\right\}
$$

where $C_{1}>0$ is so far arbitrary. So:

$$
1-d(\xi)=\left(1+\frac{2 \pi k h}{a}-\frac{2 i}{a} h^{1-1 / s}+h \tilde{\zeta}\right)^{-2} e^{i a \tilde{\zeta}} \exp \left[-\left(\tilde{\eta}_{k}-\frac{2 i}{a}+h^{1 / s \tilde{\zeta}}\right)^{2} h^{\prime}\right]
$$

By a Taylor expansion, we see easily that:

$$
1-d(\xi)-e^{i a \tilde{\zeta}}=\mathcal{O}\left(h^{\prime}\right)
$$

uniformly for $\eta \in B_{k}(h)$. Now, if $|\operatorname{Re} \tilde{\zeta}|<\frac{\pi}{a}$ and $|\operatorname{Im} \tilde{\zeta}|<C_{1}$, the equation $e^{i a \tilde{\zeta}}=1$ has the unique solution $\tilde{\zeta}=0$ and this is a simple root. Moreover, it is easy to see that $1-e^{i a \tilde{\zeta}}$ is bounded away from 0 on the boundary of $|\operatorname{Re} \tilde{\zeta}|<\frac{\pi}{a}$, $|\operatorname{Im} \widetilde{\zeta}|<C_{1}$. So by Rouché's theorem, one can assert from (1.12) that equation $d(\xi)=0$ has a simple root in $B_{k}(h)$. The same holds for $d_{6}(\xi)$ and $d_{1}(\xi)$, and $d_{0}(\xi)$ has exactly 2 roots in $B_{k}(h)$. Further, all these roots depend smoothly on $h>0$, and admit clearly asymptotic expansions. Namely, when:

$$
\eta_{k}=h^{1-1 / s}\left(\tilde{\eta}_{k}-\frac{2 i}{a}\right)+h \tilde{\zeta}_{k}
$$


denotes such a root (the roots of $d_{1}(\xi), d_{6}(\xi)$ or $d_{0}(\xi)$ differ only by $\mathcal{O}\left(e^{-C / h^{1 / s}}\right)$ from these of $d(\xi))$, then we have:

$$
\widetilde{\zeta}_{k} \sim \sum_{j=1}^{\infty} h^{\prime j} \zeta_{k, j}(h)
$$

in the sense of analytic symbols in $h^{\prime}$ (see definition above), and we can choose a realization of this symbol up to $\mathcal{O}\left(e^{-C / h^{\prime}}\right)$ if $\eta_{k}$ is a root of $d(\xi)$, and up to $\mathcal{O}\left(e^{-C / h^{1 / s}}\right)$ if $\eta_{k}$ is a root of $d_{0}(\xi), d_{1}(\xi)$ or $d_{6}(\xi)$. At the level of principal symbol, we have:

$$
\zeta_{k .1}(h)=\frac{c_{k}}{i a}\left(c_{k}-2 h^{1 / s}\right)\left(1-\frac{2 c_{k}}{i a} h^{1-1 / s}-2 \frac{h}{i a}\right)^{-1}
$$

where $c_{k}=\tilde{\eta}_{k}-\frac{2 i}{a} \neq 0$. Note that it seems impossible to get rid of the $h$-dependence of $\zeta_{k, j}(h)$, as is usual with Gevrey asymptotic expansions [Le].

It is easy to strenghten the previous estimates to $H^{2}\left(\Gamma_{\theta}\right)$-norms (this amounts to introduce some negative powers of $h$, that will be damped by the exponentials). Further all our estimates equally hold for the formal adjoint $P(\xi)$ * which has the same structure. So far, we have proved the following standard Fredholm alternative for the operator $U(\xi)=I-(G(\xi) V)^{2}$ which is close to our final result:

Proposition 1.8. Let $s>3$. For $h>0$ small enough we have the following: if $a=(2 m+1) h, m \in \mathbb{N}$ in such a way that $\left|a-a_{0}\right|<$ Const. $h$ as $h \rightarrow 0$, there exists a discrete subset $\Omega_{h} \subset W_{h}$ such that:

$1^{\circ}$ If $\eta \in W_{h} \backslash \Omega_{h}$, then $U(\xi)$ is an automorphism of $L^{2}\left(\Gamma_{\theta}\right)$ and of $H^{2}\left(\Gamma_{\theta}\right)$.

$2^{\circ}$ If $\eta \in \Omega_{h}$, then $0 \in \sigma(U(\xi)), U(\xi)$ is Fredholm of index 0 and splits into a direct sum $U(\xi): \widetilde{F}(\xi) \oplus \widetilde{F^{\prime}}(\xi) \rightarrow \widetilde{F}(\xi) \oplus \widetilde{F^{\prime}}(\xi)$, where $\tilde{F}(\xi)$ is finite dimensional vector space, $\widetilde{F^{\prime}}(\xi)$ is closed, $U(\xi)_{\mid \widetilde{F}(\xi)}$ is nilpotent of order at most 2 , whereas $U(\xi)$ is an automorphism of $\widetilde{F^{\prime}}(\xi)$ and of $\widetilde{F^{\prime}}(\xi) \cap H^{2}\left(\Gamma_{\theta}\right)$.

$3^{\circ}$ There is a surjection $f$ from $\Omega_{h}$ onto the roots of the equation: $2 \pi h e^{-\eta^{2} / h} e^{2 i a \xi / h} \gamma(\xi)=1$ in $W_{h}$, such that $f(\eta)-\eta=\mathcal{O}\left(e^{-C / h^{1 / s}}\right), C>0$.

$4^{\circ}$ If $\eta \in \Omega_{h}$, then $\eta$ admits an expansion as a classical analytic symbol in $h^{\prime}$ given by (1.15) and (1.16) 


\section{End of the Proof}

We show that $\Omega_{h}$ is actually a set of resonances for $P$. Let $\xi_{0}$ be one of the roots of $d(\xi)=0$ in $W_{h}$. We know that there exist two roots (counted with possible multiplicity) of $d_{0}(\xi)=0$ in $\left|\xi-\xi_{0}\right|<\varepsilon h(\varepsilon>0)$ and that they are exponentially close to $\xi_{0}$ in the scale $e^{-C / h^{1 / s}}$. Consider the spectral projector for $P$ in $\left|\xi-\xi_{0}\right| \leq \varepsilon h$ :

$$
\Pi\left(\xi_{0}\right)=\frac{1}{i \pi} \oint\left(I-(G(\xi) V)^{2}\right)^{-1}(I-G(\xi) V) G(\xi) \xi d \xi
$$

where $\oint$ denotes integral along the closed loop $\left|\xi-\xi_{0}\right|=\varepsilon h$. It is easy to check that (1.8) and (1.10) hold on this loop. So we have:

$$
\left.(I-G(\xi) V)^{2}\right)^{-1}=(I+\gamma(\xi) S(\xi))(I-\gamma(\xi) H(\xi))^{-1}
$$

where:

$$
S(\xi)=\sum_{i, j=1}^{12} d_{i j}(\xi) f_{i}^{\prime}(x) g_{j}(y)
$$

and $d_{i j}(\xi)$ is given by $X_{i}=\sum_{j=1}^{12} d_{i j}(\xi) Y_{j}$. By Cauchy's formula and relation ${ }^{t} G(\xi)=G(\xi)$ :

$$
\Pi\left(\xi_{0}\right)=\frac{1}{i \pi} \oint \sum_{i, j} d_{i j}(\xi) f_{i}^{\prime}(x) A(\xi) g_{j}(y) \gamma(\xi) \xi d \xi
$$

where $A(\xi)=G(\xi)(I-V G(\xi))\left(I-\gamma(\xi)^{t} H(\xi)\right)^{-1}$. Because of $(1.6)_{i}$, Lemma 1.7 and Cauchy's formula, we get:

$$
\Pi\left(\xi_{0}\right)=\frac{1}{i \pi} \sum_{k=1}^{6} \oint F_{k}(x) A(\xi) G_{k}(y) \gamma(\xi) \xi d \xi+\mathcal{O}\left(e^{-1 / C h^{\prime}}\right)
$$

where :

$$
\begin{gathered}
F_{k}(x)=f_{k}^{\prime}(x)+\sum_{i=7}^{12} \gamma(\xi)\left\langle f_{k}^{\prime} \mid g_{i}\right\rangle f_{i}^{\prime}(x), k=1, \cdots, 6 \\
G_{k}(y)=\sum_{j=1}^{6} d_{k j}(\xi) g_{j}(y), k=1, \cdots, 6 .
\end{gathered}
$$

We shall compute $F_{k}(x)$ and $G_{k}(y)$ in the following lemmas, which we can prove by a straightforward computation. 
Lemma 2.1. For $\eta \in W_{h}$, we have:

$1^{\circ} \quad F_{1}(x)=\kappa(\xi) e^{-i x \xi / h}$

$$
\begin{aligned}
& {[(1-d(\xi)) \chi(x<-\delta)+\chi(-\delta<x<\delta)+(1-d(\xi)) \chi(\delta<x<a-\delta)]} \\
& +e^{6 b / a h^{1 / s}} \mathcal{O}(1)
\end{aligned}
$$

$2^{\circ} \quad F_{2}(x)=\chi(x<\delta) e^{i x \xi / h} \varphi_{1}(x)+e^{-h^{-1 / s}} e^{6 b / a h^{1 / s}} \mathcal{O}(1)$

$3^{\circ} \quad F_{3}(x)=e^{-i x \xi / h}[(1-d(\xi)) \chi(x<-\delta)+\chi(x<\delta)+(1-d(\xi)) \chi(\delta<x<a-\delta)]$

$$
+e^{-h^{-1 / s}} e^{6 b / a h^{1 / s}} \mathcal{O}(1)
$$

$4^{\circ} \quad F_{4}(x)=e^{i x \xi / h}[\chi(x>a-\delta)+(1-d(\xi)) \chi(\delta<x<a-\delta)$

$+(1-d(\xi)) \chi(x>a+\delta)]+\gamma(\xi) \kappa(\xi) \chi(-\delta<x<\delta) e^{-i x \xi / h} S_{-}(x)$

$+e^{-h^{-1 / s}} e^{6 b / a h^{1 / s}} \mathcal{O}(1)$

$5^{\circ} \quad F_{5}(x)=\chi(x>a-\delta) e^{-i x \xi / h} \varphi_{2}(x)+\left(\gamma(\xi) e^{2 i a \xi / h} \mathscr{O}(1)\right.$

$\left.+\gamma^{2}(\xi) e^{4 i a \xi / h} \mathcal{O}(1)\right) \chi(x<a-\delta) e^{-i x \xi / h}+e^{-h^{-1 / s}} e^{6 b / a h^{1 / s}} \mathcal{O}(1)$

$6^{\circ} \quad F_{6}(x)=\sqrt{2 \pi h} e^{-\eta^{2} / 2 h} e^{i x \xi / h}[(1-d(\xi)) \chi(\delta<x<a-\delta)$

$+\chi(a-\delta<x<a+\delta)+(1-d(\xi)) \chi(x>a+\delta)]$

$+\chi(-\delta<x<\delta) e^{-i x \xi / h} S_{-}(x)+e^{-h^{-1 / s}} e^{6 b / a h^{1 / s}} \mathcal{O}(1)$

Now we compute the $G_{k}(y)$ 's. We put:

$$
\begin{aligned}
& \tilde{\varphi}_{1}(x)=\left(\sqrt{2 \pi h} e^{-\eta^{2} / 2 h}\right)^{-1} \varphi_{1}(x) \\
& \tilde{\varphi}_{2}(x)=\kappa(\xi)^{-1} \varphi_{2}(x)=\tilde{\varphi}_{1}(a-x)
\end{aligned}
$$

Functions $\tilde{\varphi}_{1}$ and $\tilde{\varphi}_{2}$ are "normalized" in the sense:

$$
\tilde{\varphi}_{1}(x), \tilde{\varphi}_{2}(x)=1+\mathcal{O}\left(e^{-1 / C h^{\prime}}\right), \quad \delta<x<a-\delta
$$

We introduce again some notations. By $\operatorname{Hol}(y, \xi)$ we mean any holomorphic function $g(y, \xi, h)$ of $\xi \in W_{h}$ with temperate growth in the scales $\exp c h^{1 / s}$, and which decays as fast as $e^{-y^{2} / 2 h}+e^{-(y-a)^{2} / 2 h}$ with respect to $y \in \mathbf{R}$. $\operatorname{By} \operatorname{Mer}(y, \xi)$ we mean any meromorphic function $g(y, \xi, h)$ of $\xi \in W_{h}$ whose numerator is $\left(e^{-y^{2} / 2 h}+e^{-(y-a)^{2} / 2 h}\right) e^{12 b / a h^{1 / s}} \mathcal{O}(1)$, and whose denominator is of the form: $d_{6}(\xi)^{k} d_{1}(\xi)^{l}$, with $k, l \in \mathbf{N}, 1 \leq k+l \leq 2$. We have: 
Lemma 2.2. For $\left|\xi-\xi_{0}\right|=\varepsilon h$, we have:

$1^{\circ} G_{1}(y)=\frac{1}{d_{1}(\xi)} e^{i y \xi / h} \psi_{-}(y)\left[\chi(-\delta<y<\delta)+(1-d(\xi)) \chi(a-\delta<y<a+\delta) \tilde{\varphi}_{2}(y)\right]$ $+e^{-2 h^{-1 / s}} \operatorname{Mer}(y, \xi)$

$2^{\circ} \quad G_{2}(y)=\frac{1}{d_{6}(\xi)} e^{-i y \xi / h} \psi_{+}(y)\left[(1-d(\xi))^{2} \chi(-\delta<y<\delta) \tilde{\varphi}_{1}(y)\right.$

$$
+(1-d(\xi)) \chi(a-\delta<y<a+\delta)]+\operatorname{Hol}(y, \xi)+e^{-h^{-1 / s}} \operatorname{Mer}(y, \xi)
$$

$3^{\circ} \quad G_{3}(y)=\frac{1}{d_{1}(\xi)} \gamma^{2}(\xi) \kappa^{2}(\xi) \mathcal{O}(1) \chi(y<\delta) e^{i y \xi / h} \psi_{-}(y)$

$$
+\frac{1}{d_{1}(\xi)} \gamma^{3}(\xi) \kappa^{3}(\xi) \mathcal{O}(1) \chi(y>a-\delta) e^{i y \xi / h} \psi_{-}(y) \tilde{\varphi}_{2}(y)+\operatorname{Hol}(y, \xi)+e^{-h^{-1 / s}} \operatorname{Mer}(y, \xi)
$$

$4^{\circ} \quad G_{4}(y)=\operatorname{Hol}(y, \xi)+e^{-h^{-1 / s}} \operatorname{Mer}(y, \xi)$

$5^{\circ} \quad G_{5}(y)=\frac{1}{d_{1}(\xi)} e^{i y \xi / h} \psi_{-}(y)\left[(1-d(\xi)) \chi(-\delta<y<\delta)+(1-d(\xi))^{2}\right.$

$$
\left.\chi(a-\delta<y<a+\delta) \tilde{\varphi}_{2}(y)\right]+\operatorname{Hol}(y, \xi)+e^{-2 h^{-1 / s}} \operatorname{Mer}(y, \xi) .
$$

$6^{\circ} \quad G_{6}(y)=\frac{1}{d_{6}(\xi)} e^{-i y \xi / h} \psi_{+}(y)\left[(1-d(\xi)) \chi(-\delta<y<\delta) \tilde{\varphi}_{1}(y)+\chi(a-\delta<y<a+\delta)\right]$

$+e^{-h^{-1 / s}} \operatorname{Mer}(y, \xi)$

where all estimates are uniform on a neighborhood of $\left|\xi-\xi_{0}\right|=\varepsilon h$.

Replace $d_{1}(\xi)$ and $d_{6}(\xi)$ by $d(\xi)$ in the formulae giving $G_{k}(y)$ and denote by $\frac{1}{d(\xi)} \tilde{G}_{k}(y)$ the meromorphic part, modulo $e^{-h^{-1 / s}} \operatorname{Mer}(y, \xi)$, of the corresponding functions. Since $d_{1}(\xi) \equiv d_{6}(\xi) \equiv d(\xi) \bmod \mathcal{O}\left(e^{-1 / C h^{1 / s}}\right)$, and $|d(\xi)| \geq$ Cst on $\left|\xi-\xi_{0}\right|$ $=\varepsilon h$, we get:

$$
\begin{aligned}
\left.\tilde{G}_{1}(y)\right|_{\xi=\xi_{0}} & =\left.\tilde{G}_{5}(y)\right|_{\xi=\xi_{0}} \\
& =e^{i y \xi / h} \psi_{-}(y)\left[\chi(-\delta<y<\delta)+\left.\chi(a-\delta<y<a+\delta) \tilde{\varphi}_{2}(y)\right|_{\xi=\xi_{0}}\right. \\
\left.\tilde{G}_{2}(y)\right|_{\xi=\xi_{0}} & =\left.\tilde{G}_{6}(y)\right|_{\xi=\xi_{0}} \\
& =e^{-i y \xi / h} \psi_{+}(y)\left[\chi(-\delta<y<\delta) \tilde{\varphi}_{1}(y)+\left.\chi(a-\delta<y<a+\delta)\right|_{\xi=\xi_{0}}\right.
\end{aligned}
$$

and $\left.\widetilde{G}_{4}(y)\right|_{\xi=\xi_{0}}=0$. So we define the outgoing functions : 


$$
\begin{aligned}
& F_{+}(x)=F_{2}(x)+\left.F_{6}(x)\right|_{\xi=\xi_{0}} \\
& \left.=\sqrt{2 \pi h} e^{-\eta^{2} / 2 h} e^{i x \xi / h}\left[\chi(-\delta<x<\delta) \tilde{\varphi}_{1}(x)+\chi(x>\delta)\right)\right]\left.\right|_{\xi=\xi_{0}} \\
& +\left.\chi(-\delta<x<\delta) e^{-i x \xi / h} S_{-}(x)\right|_{\xi=\xi_{0}}+e^{-h^{-1 / s}} e^{6 b / a h^{1 / s}} \mathcal{O}(1) \\
& F_{-}(x)=F_{1}(x)+\left.F_{5}(x)\right|_{\xi=\xi_{0}} \\
& =\left.\kappa(\xi) e^{-i x \xi / h}\left[\chi(x<a-\delta)+\chi(a-\delta<x<a+\delta) \tilde{\varphi}_{2}(x)\right]\right|_{\xi=\xi_{0}} \\
& +e^{6 b / a h^{1 / s}} \mathcal{O}(1) \text {. }
\end{aligned}
$$

Note that $F_{+}(x)$ and $\frac{1}{\kappa(\xi)} F_{-}(x)$ are outgoing except microlocally near the points $\left(0, \pm \frac{1}{2}\right),\left(a, \pm \frac{1}{2}\right)$. Now we apply the formula of residues to (2.2). The analysis of the determinants carried on in Sect. 1.4 shows that (1.8) and (1.10) are fulfilled and we get:

$$
\begin{aligned}
\Pi\left(\xi_{0}\right)= & \frac{2}{\dot{d}\left(\xi_{0}\right)} \gamma\left(\xi_{0}\right) \xi_{0}\left[\left.F_{-}(x) A(\xi) \tilde{G}_{1}(y)\right|_{\xi=\xi_{0}}+\left.F_{+}(x) A(\xi) \tilde{G}_{2}(y)\right|_{\xi=\xi_{0}}\right. \\
& \left.+\left.F_{3}(x) A(\xi) \tilde{G}_{3}(y)\right|_{\xi=\xi_{0}}+\left.F_{4}(x) A(\xi) \tilde{G}_{4}(y)\right|_{\xi=\xi_{0}}\right]+\mathcal{O}\left(e^{-1 / C h^{1 / s}}\right)
\end{aligned}
$$

where the dot denotes derivative with respect to $\xi$. Thus there remains to compute $\left.A(\xi) \tilde{G}_{j}(y)\right|_{\xi=\xi_{0}}, j=1, \cdots, 4$. First Lemma 1.3 gives $L^{2}\left(\Gamma_{\theta}\right)$-estimates:

$$
\begin{aligned}
& \left.\tilde{G}_{1}(y)\right|_{\xi=\xi_{0}},\left.\quad \tilde{G}_{3}(y)\right|_{\xi=\xi_{0}}=\mathcal{O}(1) \\
& \left.\tilde{G}_{2}(y)\right|_{\xi=\xi_{0}}=e^{-a \operatorname{Im} \eta / 2 h} \mathcal{O}(1)
\end{aligned}
$$

while (the proof of ) Lemma 1.1 and 1.2 yields:

$$
\left(I-\gamma(\xi)^{t} H(\xi)\right)^{-1}=I+\gamma(\xi)^{t} K^{\prime}(\xi)+e^{-2 h^{-1 / s}} e^{b / a h^{1 / s}} \mathcal{O}(1)
$$

and

$$
{ }^{t} K^{\prime}(\xi)=e^{-a \operatorname{Im} \eta / 2 h} e^{b / a h^{1 / s}} \mathcal{O}(1)
$$

as bounded operators on $L^{2}\left(\Gamma_{\theta}\right)$. An easy computation gives the kernel of $G(\xi) V G(\xi)$ which we write as :

$$
\begin{aligned}
& G(\xi) V G(\xi)(t, y)=C(h)\left[\beta(t, x) e^{i(x-t) \xi / h}+\beta(x, t) e^{-i(x-t) \xi / h}\right. \\
& \left.+\int_{-\infty}^{t \wedge y} \psi_{-}(y) d y+\int_{t \vee y}^{\infty} \psi_{+}(y) d y\right]
\end{aligned}
$$

where we have omitted as before, the indices $\theta$ referring to distorsion, and $C(h)=-(2 \xi h)^{-2} \sqrt{h / 2 \pi} e^{-h^{-1 / s}}$. For $\xi=\xi_{0}$ we readily find : 


$$
\begin{aligned}
& G(\xi)=e^{-a \operatorname{Im} \eta / 2 h} e^{b / a h^{1 / s}} \mathcal{O}(1) \\
& G(\xi) V G(\xi)=e^{-a \operatorname{Im} \eta / 2 h} \mathcal{O}(1)
\end{aligned}
$$

as bounded operators on $L^{2}\left(\Gamma_{\theta}\right)$. Relations (2.6)-(2.9) show that the main contribution to $\left.A(\xi) \tilde{G}_{1}(y)\right|_{\xi=\xi_{0}}$ is given by $\left.G(\xi)(I-V G(\xi)) \tilde{G}_{1}(y)\right|_{\xi=\xi_{0}}$. Explicitely, after some straight-forward computation, we find:

$$
\begin{aligned}
\left.\tilde{G}_{1}(y)\right|_{\xi=\xi_{0}}= & i(2 \xi h)^{-1} \sqrt{2 \pi h} e^{-\eta^{2} / 2 h} e^{i y \xi / h}[\chi(\delta<y<a-\delta)+\chi(y>a+\delta)] \\
& +\chi(-\delta<y<\delta) \mathcal{O}(1)+\chi(a-\delta<y<a+\delta) \mathcal{O}(1)+\mathcal{O}\left(e^{-1 / C h^{1 / s}}\right)
\end{aligned}
$$

where the terms $\mathcal{O}(1)$ are microlocally concentrated near $\xi=\frac{1}{2}, \frac{-3}{2}$. So $\left.\widetilde{G}_{1}(y)\right|_{\xi=\xi_{0}}$ is microlocally concentrated near $[0,+\infty[\cup($ finite set $)$, with amplitude $\mathcal{O}(1)$. Similarly,

$$
\begin{aligned}
& \left.\tilde{G}_{2}(y)\right|_{\xi=\xi_{0}}=i(2 \xi h)^{-1} \kappa(\xi) e^{-i y \xi / h}[\chi(y<-\delta)+\chi(a-\delta<y<a+\delta)]+ \\
& \kappa(\xi) \chi(-\delta<y<\delta) \mathcal{O}(1)+\kappa(\xi) \chi(a-\delta<y<a+\delta) \mathcal{O}(1)+\kappa(\xi) \mathcal{O}\left(e^{-1 / C h^{1 / 5}}\right)
\end{aligned}
$$

where the terms $\mathcal{O}(1)$ are microlocally concentrated near $\xi=\frac{-1}{2}, \frac{3}{2}$. So $\left.\tilde{G}_{2}(y)\right|_{\xi=\xi_{0}}$ is microlocally concentrated near $]-\infty, \mathrm{a}] \cup($ finite set), with amplitude $\kappa(\xi) \mathcal{O}(1)$. Moreover, it is easy to see that $\left.F_{3}(x) A(\xi) \tilde{G}_{3}(y)\right|_{\xi=\xi_{0}}$ and $F_{4}(x) A(\xi)$ $\left.\widetilde{G}_{4}(y)\right|_{\xi=\xi_{0}}$ can be neglected in their contribution to $\Pi\left(\xi_{0}\right)$. So we have, putting $G_{-}(y)=\left.A(\xi) \tilde{G}_{1}(y)\right|_{\xi=\xi_{0}}$ and $G_{+}(y)=\left.A(\xi) \tilde{G}_{2}(y)\right|_{\xi=\xi_{0}}$ :

$$
\Pi\left(\xi_{0}\right)=\frac{2}{\dot{d}\left(\xi_{0}\right)} \gamma\left(\xi_{0}\right) \xi_{0}\left[F_{+}(x) G_{+}(y)+F_{-}(x) G_{-}(y)\right]+\mathcal{O}\left(e^{-C / h^{1 / s}}\right)
$$

and the two terms inside the bracket have same amplitude $\sim \kappa\left(\xi_{0}\right)$. The projector $\Pi\left(\xi_{0}\right)$ is of rank at least 2 . Our purpose was to solve the eigenvalue problem $P u=-h^{2} u^{\prime \prime}+V(x, h) u=\xi^{2} u$ ou $L^{2}\left(\Gamma_{\theta}\right)$ for $\xi$ close to $\frac{1}{2}$. We know from the general theory of ordinary differential equations that the eigenspace corresponding to a resonance $\xi^{2}$ is one dimensional. Namely, for given $\xi$, $\operatorname{Re} \xi>0$, let $u_{ \pm}(x)$ be the solution of $\left(P-\xi^{2}\right) u=0$ in $L^{2}\left(\Gamma_{\theta}\right)$, with $u_{ \pm} \sim e^{ \pm i x \xi / h}$, $x \rightarrow \pm \infty$ (i.e. $u_{+}$is outgoing at $+\infty, u_{-}$is outgoing at $-\infty$ ); then $\xi^{2}$ is a resonance iff $u_{+}$and $u_{-}$are colinear. But as $P$ is not self adjoint, it cannot be excluded that $\xi^{2}$ is a multiple pole for the resolvent $\left(P-\xi^{2}\right)^{-1}$; in that case, stability arguments (see [Ra] and also [Sj2], [KaRo] for related results) show that this multiplicity is at most 2 . So we can conclude that the eigenprojector $\Pi\left(\xi_{0}\right)$ is of rank exactly 2 and that the alternative of Theorem $0.1,3^{\circ}$ holds. The 
other statements easily follow from Proposition 1.8. So we have proved the Theorem.

Q.E.D.

\section{Appendix}

Proof of Lemma 1.1. The error $H^{\prime}(\xi)$ consists in: 1) replacing $\varphi_{1}(x \wedge y)$ and $\varphi_{2}(x \vee y)$ by constant values near and $(0,0)$ and $\left.(a, a) ; 2\right)$ ignoring the contribution to $K(\xi)$ of a neighborhood of the diagonal outside these points ; 3 ) omitting some products $f_{j}(x) g_{j}(y)$, where $f_{j}(x)$ 's and $g_{j}(y)$ 's range among $\langle 2+|$, $\langle 6+|,\langle 8-|,\langle 12-|$ and $|+2\rangle,|+6\rangle,|-8\rangle,|-12\rangle$ respectively (the definition of these functions is clear from the expression of $K(\xi)$.) Consider first :

$$
\begin{gathered}
H_{6}(\xi)=\chi((-\delta<x<\delta) \cap(-\delta<y<\delta))\left[e^{i(x-y) \xi / h} \psi_{+}(y) \varphi_{1}(x \wedge y)+e^{-i(x-y) \xi / h} \psi_{-}(y)\right. \\
\left(\varphi_{2}(x \vee y)-e^{2 i a \xi / h} e^{-\eta^{2} / 2 h} \sqrt{2 \pi h)}\right]
\end{gathered}
$$

By (1.2) we have, for $z \in[-\delta, \delta], \alpha\left(a-z, \eta^{\prime}\right)=\mathcal{O}\left(e^{-1 / C h}\right), \alpha(a-z, \eta)=e^{-\eta^{2} / 2 h} \sqrt{2 \pi h}+$ $\mathcal{O}\left(e^{-1 / C h}\right)$. On the other hand,

$$
\alpha\left(-z, \eta^{\prime}\right) \leq e^{(\operatorname{Im} \eta)^{2} / 2 h} \int_{-\infty}^{-z} e^{-(t-\operatorname{Im} \eta)^{2} / 2 h} d t
$$

Since $\operatorname{Im} \eta=\mathcal{O}\left(h^{1-1 / s}\right)$ and $s \geq 2$ we get $\alpha\left(-z, \eta^{\prime}\right)=\mathcal{O}(1)$; the same holds for $\alpha(-z, \eta)$. Moreover for $x, y \in[-\delta, \delta]$ we have $e^{-i(x-y) \xi / h} \psi_{-}(y)=\mathcal{O}(1)$. Collecting these estimates gives:

$$
e^{-i(x-y) \xi / h} \psi_{-}(y) \varphi_{2}(x \vee y)-e^{2 i a \xi / h} e^{-\eta^{2} / 2 h} \sqrt{2 \pi h}=\mathcal{O}(1)
$$

A similar argument leads to:

$$
e^{i(x-y) \xi / h} \psi_{+}(y) \varphi_{1}(x \wedge y)=\mathcal{O}(1)
$$

and finally $H_{6}(\xi)=\mathcal{O}(1)$. We have the same estimate for the term $H_{8}(\xi)$ localized near $(a, a)$, and an $L^{2}$ estimate for the corresponding operators. Consider now, on one side of the diagonal,

$$
H_{3}(\xi)=\chi(x<y<-\delta)\left[e^{i(x-y) \xi / h} \psi_{+}(y) \varphi_{1}(x)+e^{-i(x-y) \xi / h} \psi_{-}(y) \varphi_{2}(y)\right]
$$

where we have omitted the subscript $\theta$ referring to distorsion. By Schur's lemma we need estimate:

$$
\sup _{x<-\delta} \int_{x}^{-\delta}\left|H_{3}(\xi)\right| d y, \quad \sup _{y<-\delta} \int_{-\infty}^{y}\left|H_{3}(\xi)\right| d x
$$


For $-b<x<-\delta$, we may replace the first quantity in (a.1) by $\sup _{-b<x, y<-\delta}$ $\left|H_{3}(\xi)\right|=\mathcal{O}\left(e^{-1 / C h^{\prime}}\right)$. Write $H_{3}(\xi)=H_{3}^{+}(\xi)+H_{3}^{-}(\xi)$. We analyse $H_{3}^{-}(\xi)$ first. We have:

$$
\begin{aligned}
\alpha\left(-y_{\theta}, \tau\right)= & \alpha\left(-\operatorname{Re} y_{\theta}, \tau\right) \\
& -i \operatorname{Im} y_{\theta} e^{-\tau^{2} / 2 h} \int_{0}^{1} \exp \left[-\left(\operatorname{Re} y_{\theta}+i t \operatorname{Im} y_{\theta}-i \tau\right)^{2} / 2 h\right] d t
\end{aligned}
$$

It is readily seen that for $\theta>0$ small enough (depending on $b$ only) we have:

$$
\begin{aligned}
& \alpha\left(-y_{\theta}, \eta\right), \quad \alpha\left(a-y_{\theta}, \eta\right)=\sqrt{2 \pi h} e^{-\eta^{2} / 2 h}+\mathcal{O}\left(e^{-1 / C h^{\prime}}\right), \quad y<-\delta \\
& \alpha\left(-y_{\theta}, \eta^{\prime}\right), \quad \alpha\left(a-y_{\theta}, \eta^{\prime}\right)=\mathcal{O}\left(e^{-1 / C h^{\prime}}\right), \quad y<-\delta
\end{aligned}
$$

all estimates being uniform on the given intervals. Next we have:

$$
\psi_{ \pm}\left(y_{\theta}\right)=e^{-y_{\theta}^{2} / 2 h}\left(e^{ \pm i y_{\theta} \eta}+e^{ \pm i y_{\theta} \eta^{\prime} / h}\right)+\mathcal{O}\left(e^{-\left(y_{\theta}-a\right)^{2} / 2 h}\right), \quad y<-\delta
$$

so:

$$
\begin{aligned}
\left|\psi_{ \pm}\left(y_{\theta}\right)\right| \leq & C \exp \left(-y^{2} \cos 2 \theta(y) / 2 h\right) \\
& \times\left(\exp \left(\mp y \operatorname{Im}\left(\eta e^{i \theta(y)}\right) / h\right)+\exp \left(\mp y \operatorname{Im}\left(\eta^{\prime} e^{i \theta(y)}\right) / h\right), \quad y<-\delta\right.
\end{aligned}
$$

From these estimates, it follows easily that if $\theta>0$ is small enough, we have:

$$
\sup _{x<-\delta} \int_{x}^{-\delta}\left|H_{3}^{-}(\xi)\right| d y=\mathcal{O}\left(e^{-1 / C h^{\prime}}\right)
$$

For the integral with respect to $x$, we use also that $\left|e^{-i x_{\theta} \xi / h}\right|$ is integrable when $x \rightarrow-\infty$. So: $\sup _{y<-\delta} \int_{-\infty}^{y}\left|H_{3}^{-}(\xi)\right| d x=\mathcal{O}\left(e^{-1 / C h^{\prime}}\right)$. Now consider $H_{3}^{+}(\xi)$. The result follows this time from the exponential decrease of $\varphi_{1}\left(x_{\theta}\right)$. Namely, by (a.2) we have:

$$
\begin{aligned}
\alpha\left(x_{\theta}, \tau\right)= & \int_{-\infty}^{\operatorname{Re} x_{\theta}} e^{-t^{2} / 2 h} e^{i t \tau / h} d t+i \operatorname{Im} x_{\theta} e^{-\tau^{2} / 2 h} \\
& \times \int_{0}^{1} \exp \left[-\left(\operatorname{Re} x_{\theta}+i t \operatorname{Im} x_{\theta}+i \tau\right)^{2} / 2 h\right] d t
\end{aligned}
$$

so when $\theta>0$ is small enough:

$$
\alpha\left(x_{\theta}, \tau\right)=e^{-x^{2} / 4 h} \mathcal{O}(1), \quad x<-\delta
$$


with uniform estimate on $x<-\delta$, with ensures integrability. The exponential decrease, again is given by $\psi_{+}\left(y_{\theta}\right)$. Thus we have $H_{3}(\xi)=\mathcal{O}\left(e^{-1 / C h}\right)$ in operator norm $L^{2}\left(\Gamma_{\theta}\right) \rightarrow L^{2}\left(\Gamma_{\theta}\right)$, when the distorsion is suitably chosen and $h>0$ is sufficiently small. The term $H_{4}(\xi)$ on the other side of the diagonal is treated similarly, as well as all other terms entering $H^{\prime}(\xi)$. Thus we have proved the lemma.

Q.E.D.

Proof of Lemma 1.2. We have:

$$
K^{\prime}(\xi)=e^{i\left(x_{\theta}+y_{\theta}\right) \xi / h} \psi_{-}\left(y_{\theta}\right) \beta\left(y_{\theta}, x_{\theta}\right)+e^{-i\left(x_{\theta}+y_{\theta}\right) \xi / h} \psi_{+}\left(y_{\theta}\right) \beta\left(x_{\theta}, y_{\theta}\right)
$$

We need first estimate $\beta\left(y_{\theta}, x_{\theta}\right)$; as $\tilde{V}(t)$ is a sum of terms like $e^{-1 / 2 h} e^{-(t \pm i) 2 / 2 h}$ and $e^{-1 / 2 h} e^{-(t-a \pm i)^{2} / 2 h}$ we can compute $\beta\left(x_{\theta}, y_{\theta}\right)$ by integrating the function $e^{-z^{2} / 2 h}$ along suitable polygons. For instance:

$$
\begin{aligned}
& \int_{x_{\theta}}^{y_{\theta}} e^{-(t \pm i)^{2} / 2 h} d t=\int_{\operatorname{Re} x_{\theta}}^{\operatorname{Re} \theta} e^{-t^{2} / 2 h} d t \\
& -i(x \sin \theta(x) \pm 1) \int_{0}^{1} \exp \left[-(x \cos \theta(x)+i t(x \sin \theta(x) \pm 1))^{2} / 2 h\right] d t \\
& \quad+i(y \sin \theta(y) \pm 1) \int_{0}^{1} \exp \left[-(y \cos \theta(y)+i t(y \sin \theta(y) \pm 1))^{2} / 2 h\right] d t
\end{aligned}
$$

So if $\theta>0$ is small enough, we have, uniformly along $\Gamma_{\theta}$ :

$$
\begin{aligned}
\left|\beta\left(y_{\theta}, x_{\theta}\right)\right| \leq & C \chi(y<x)\left(e^{-y^{2} / C h}+e^{-(y-a)^{2} / C h}\right. \\
& \left.+e^{-x^{2} / C h}+e^{-(x-a)^{2} / C h}+e^{-1 / 2 h}\right) .
\end{aligned}
$$

Look first at $-b<x, y<a+b$, that is the case without distorsion. Using (a.12) and similar estimates on $\psi_{1}(y)$ and $\psi_{2}(y)$ we get:

$$
K^{\prime}(\xi)=\mathcal{O}\left(\exp -\frac{a+b}{2} \operatorname{Im} \eta / h\right), \quad-b<x, y<a+b .
$$

Now we apply distorsion outside $-b<x, y<a+b$. As above, Schur's lemma, together with the above estimates, show easily that (a.13) holds in the sense of operator norm in $L^{2}\left(\Gamma_{\theta}\right)$, when the constant $b$ on the right hand side, is replaced by any $b^{\prime}>b$.

Q.E.D.

Lemma a.1. Let $\tau= \pm \eta^{\prime}$. Then there exists a classical analytic symbol 
$a(x, h) \sim \sum_{k \geq 0} a_{k}(x) h^{k}$ defined in a neighborhood of 0 such that:

$$
\alpha(x, \tau)=h e^{-x^{2} / 2 h} e^{-i x \tau / h} a(x, h)+\mathcal{O}\left(e^{-1 / C h}\right), \quad C>0 .
$$

Proof. This follows from repeated integrations by parts since the phase is non stationary, and from the fact that $\operatorname{Im} \tau=\mathcal{O}\left(h^{1-1 / s}\right)$.

Q.E.D.

Lemma a.2. There exist classical analytic symbols $a^{ \pm}(x, h) \sim \sum_{k \geq 0} a_{k}^{ \pm}(x) h^{k}$ defined in a neighborhood of 0 such that:

$$
\begin{aligned}
\int_{x}^{y} e^{-t^{2} / 2 h} \cos \frac{t}{h} d t= & h e^{-x^{2} / 2 h} e^{ \pm i x / h} a^{ \pm}(x, h) \\
& +h e^{-y^{2} / 2 h} e^{ \pm i y / h} a^{ \pm}(y, h)+\mathcal{O}\left(e^{-1 / C h^{\prime}}\right)
\end{aligned}
$$

(again the same letter a denotes several symbols, and we sum over \pm .)

Proof. By Cauchy's formula we have, for instance:

$$
\begin{aligned}
\int_{x}^{y} e^{-t^{2} / 2 h} e^{-i t / h} d t= & -e^{-1 / 2 h} \int_{x}^{y} e^{-t^{2} / 2 h} d t-i e^{-1 / 2 h} \int_{0}^{1} e^{-(x+i t)^{2} / 2 h} d t \\
& +i e^{-1 / 2 h} \int_{0}^{1} e^{-(y+i t)^{2} / 2 h} d t
\end{aligned}
$$

Consider $e^{-1 / 2 h} \int_{0}^{1} e^{-(x+i t)^{2} / 2 h} d t=e^{-x^{2} / 2 h} e^{-i x / h} \int_{0}^{1} e^{i x t / h} e^{-t(2-t) / 2 h} d t$. Introduce $\chi \in G_{0}^{s /(s-2)}(\mathbf{R})$ equal to 1 near 0 , and to 0 near 1 . We have:

$$
\int_{0}^{1} e^{i x t / h} e^{-t(2-t) / 2 h} d t=\int_{0}^{1} e^{i x t / h} e^{-t(2-t) / 2 h} \chi(t) d t+\mathcal{O}\left(e^{-1 / C h}\right)
$$

The phase $\varphi(t)=x t+i t(2-t) / 2$ is non-stationary near 0 ; so after const. $/ h^{\prime}$ integrations by parts we get:

$$
\int_{0}^{1} e^{i \varphi(t) / h} \chi(t) d t=h a(x, h)+\mathcal{O}\left(e^{-1 / C h^{\prime}}\right)
$$

where $a(x, h)$ is the realization of a classical analytic symbol. As $\int_{x}^{y} e^{-t^{2} / 2 h} e^{-i t / h} d t$ is a linear combination of such terms, we eventually get the Lemma. Q.E.D. 
Lemma a.3. When $\tau \neq 0, \operatorname{Im} \tau=\mathcal{O}\left(h^{1-1 / s}\right)$ we have:

$$
\begin{gathered}
\int_{-\delta}^{x} e^{-y^{2} / 2 h} e^{i \tau y / h} \alpha(y, \eta) a(y, h) d y=e^{-x^{2} / h} e^{i(\tau-\eta) x / h} b(x, h) \\
+e^{-x^{2} / 2 h} e^{i \tau x / h} \alpha(x, h) c(x, h)+\mathcal{O}\left(e^{-1 / C h^{\prime}}\right)
\end{gathered}
$$

where $b(x, h)$ and $c(x, h)$ are classical analytic symbols defined in a neighborhood of 0 .

Proof. Denote by $L a(x, h)$ the left hand side of (a.14), and set $\varphi(y, t)=i t^{2} / 2+i y^{2} / 2-t \eta+y \tau$. We have $\partial_{y} \varphi(y, t)=i y+\tau \neq 0$, and

$$
L a(x, h)=-\frac{h}{i} \int_{-\delta}^{x} \frac{a(y, h)}{i y+\tau}\left(\partial_{y} \int_{-\delta}^{y} e^{i \varphi(y, t) / h} d t-e^{i \varphi(y, y) / h}\right) d y
$$

Integrating by parts, we find:

$$
\begin{aligned}
L a(x, h)= & -\frac{h}{i} \int_{-\delta}^{x} \frac{a(y, h)}{i y+\tau} e^{-y^{2} / h} e^{i(\tau-\eta) y / h} d y \\
& +\frac{h}{i} \frac{a(x, h)}{i x+\tau} e^{-x^{2} / 2 h} e^{i \tau x / h} \alpha(x, \eta)-\frac{h}{i} L a_{1}(x, h)
\end{aligned}
$$

where $L a_{1}(x, h)$ is of the same form as $L a(x, h)$ for $a_{1}(y, h)=\partial_{y}\left(\frac{a(y, h)}{i y+\tau}\right)$. Iterating this procedure const./ $h^{\prime}$ times (we could do it const./ $h$ times, which would give a smaller remainder, but we do not need it ) and using simple induction arguments based on Cauchy inequalities, we are led to (a.14). Q.E.D.

Remark a.4. Simple variants of this Lemma are also needed, e.g. replacing the integral over $[-\delta, x]$ by an integral over $[x, \delta], \alpha(y, \eta)$ by $\alpha^{2}(y, \eta)$, etc...

\section{References}

[BaLeRa] Bardos, C., Lebeau, G. and Rauch, J., Scattering frequencies and Gevrey 3 singularities, Inv. Math., 90 (1987), 77-114.

[BrCoDu] Briet, Ph., Combes, J. M. and Duclos, P., On the location of resonances for Schrödinger operators in the semi-classical limit $1^{\circ}$ Resonance free domains, $J$. Math. Anal. Appl., 126 (1987), 90-99. $2^{\circ}$ Comm. Part. Diff. Eq., 12 (1987), $201-222$.

[CoDuKleSe] Combes, J. M., Duclos, P., Klein, M. and Seiler, R., The Shape Resonance, Comm. Math. Phys., 110 (1987), 215-230.

[CFKS] Cycon, A. L., Froese, F. G., Kirsch, W. and Simon, B., Schrödinger Operators, Springer, 1987. 
[Ge] Gérard, C., Asymptotique des pôles de la matrice de scattering pour obstacles strictement convexes. Bull. Soc. Math. France, Mémoire no 31, 116 (1) (1988).

[GeSj] Gérard, C. and Sjöstrand, J., Semi-classical resonances generated by a closed trajectory of hyperbolic type, Comm. Math. Phys., 108 (1987), 391-421.

[GuSt] Guillemin, V. and Sternberg, S., Geometric Asymptotics, Math. Surveys Monographs, 14 (1977).

[HeSj] Helffer, B. and Sjöstrand, J., Resonances en limite semi-classique, Bull. Soc. Math. France., Mémoire $\mathrm{n}^{\circ}$ 24/25 1143 (1986).

[HiSi] Hislop, P. and Sigal, I. M., Semi-classical theory of shape resonances in Quantum Mechanics, Mem. A.M.S., 78399 (1989).

[Hu] Hunziker, W. Distortion analyticity and molecular resonances curves, Ann. I.H.P., Sect. Phys. Théor., 454 (1986), 339-358.

[Ik] Ikawa, M., $1^{\circ}$ On the poles of the scattering matrix for 2 stricty convex obstacles, J. Math. Kyoto Univ., 23 (1983), 127-194. $2^{\circ}$ On the existence of poles of the scattering matrix for several convex bodies, Proc. Japan Acad. Ser. A. Math. Sc., 64 (1988), 91-93

[KaRo] Kaïdi, N. and Rouleux, M., Multiple resonances in the semi-classical limit, Comm. Math. Phys., 133 (1990), 617-634.

[Ki] Klaus, M., Asymptotic behaviour of Jost functions near resonance points for Wigner Von Neumann type potentials, J. Math. Phys., 32 (1991), 163-174.

$[\mathrm{Ku}]$ Kurasov. D., Zero-range potentials with internal structures and the inverse scattering problem, Lett. Math. Phys., 25 (1992), 287-297.

[LasLas] Lascar, B. and Lascar, R., Propagation des singularités Gevrey pour la diffraction. Comm. Part. Diff. Eq., 16 4,5 (1991), 547-584.

[LPh] Lax, P. and Phillips, R. Scattering theory, (revised ed.) Academic Press, 1989.

[Le] Lebeau, G., Propagation des singularités Gevrey pour le problème de Dirichlet, Proc. NATO ASI ou "Advances in Microlocal Analysis", D. Reidel (1985), 203-223.

[Me] Melrose, R., $1^{\circ}$ Singularities and energy decay in acoustical scattering, Duke Math. J., 46(1) (1979), 43-59. $2^{\circ}$ Geometric scattering theory, Cambridge Univ. Press, 1995.

[Na] Nakamura, S., $1^{\circ}$ Shape resonances for distorition analytic Schrödinger Operators, Comm. Part. Diff. Eq., 14 (1989), 1385-1419; $2^{\circ}$ Semi-classical resolvent estimates for the barrier top energy, Comm. Part. Diff. Eq., 16 (1991), 873-883.

[PetSt] Petkov, V. and Stoyanov, L., Geometry of reflecting rays and inverse spectral problems, John Wiley, 1992.

[Ra] Ramm, A. G., Perturbation of resonances, J. Math. Anal. Appl., 88 (1982), $1-7$.

[ReSim] Reed, M. and Simon, B., Methods of modern mathematical physics IV, Acad. Press, 1978.

[RiN] Riesz, F. and Sz. B., Nagy, Lecons d'analyse fonctionnelle, Gauthier Villars (1975).

[Ro] Rouleux, M., Resonance free domains for semi-classical Schrödinger operators of Gevrey type, Hokkaido Math. J. to appear.

[Sj] Sjöstrand, J., $1^{\circ}$ Singularités analytiques microlocales, Astérisque, 95 (1982) $2^{\circ}$ Semi-classical resonances generated by non degenerate critical points, Lect. Notes Math. Springer, 1256 (1987), 402-429.

[SiZw] Sjöstrand, J. and Zworski, M., Complex scaling and the distribution of scattering poles, J. Amer. Math. Soc., 4 (1991), 729-769. 
